\title{
Unsteady Mixed Convection Flow along Symmetric Wedge with Variable Surface Temperature Embedded in a Porous Medium Saturated with a Nanofluid
}

\author{
K. M. Abualnaja', M. S. Elgendy ${ }^{2}$, F. S. Ibrahim ${ }^{3}$ \\ ${ }^{1}$ Department of Mathematical Science, Faculty of Applied Science, Umm Al-Qura University, Makkah, Saudi Arabia \\ ${ }^{2}$ Department of Mathematics, University College in Jammoum, Umm Al-Qura University, Makkah, Saudi Arabia \\ ${ }^{3}$ Department of Mathematics, Faculty of Science, Assiut University, Assiut, Egypt \\ Email: fibrahim208@gmail.com
}

How to cite this paper: Abualnaja, K.M., Elgendy, M.S. and Ibrahim, F.S. (2021) Unsteady Mixed Convection Flow along Symmetric Wedge with Variable Surface Temperature Embedded in a Porous Medium Saturated with a Nanofluid. Journal of Applied Mathematics and Physics, 9, 101-126. https://doi.org/10.4236/jamp.2021.91008

Received: February 10, 2020

Accepted: January 18, 2021

Published: January 21, 2021

Copyright $\odot 2021$ by author(s) and Scientific Research Publishing Inc. This work is licensed under the Creative Commons Attribution International License (CC BY 4.0).

http://creativecommons.org/licenses/by/4.0/

\begin{abstract}
Laminar two-dimensional unsteady mixed-convection boundary-layer flow of a viscous incompressible fluid past asymmetric wedge with variable surface temperature embedded in a porous medium saturated with a nanofluid has been studied. The employed mathematical model for the nanofluid takes into account the effects of Brownian motion and thermophoresis. The velocity in the potential flow is assumed to vary arbitrary with time. The non-Darcy effects including convective, boundary and inertial effects will be included in the analysis. The unsteadiness is due to the time-dependent free stream velocity. The governing boundary layer equations along with the boundary conditions are converted into dimensionless form by a non-similar transformation, and then resulting system of coupled non-linear partial differential equations are solved by perturbation solutions for small dimensionless time until the second order. Numerical solutions of the governing equations are obtained employing the implicit finite-difference scheme in combination with the quasi-linearization technique. To validating the method used, we compared our results with previous results in earlier papers on special cases of the problem and are found to be in agreement. Effects of various parameters on velocity, temperature and nanoparticle volume fraction profiles are graphically presented.
\end{abstract}

\section{Keywords}

Nanofluids, Unsteady Flow, Mixed Convection, Boundary Layer, Wedge Flow, Finite Difference, Non-Similarity Solutions, Porous Media 


\section{Introduction}

The study of mixed convection flow finds applications in several industrial and technical processes such as nuclear reactors cooled during emergency shutdown, solar central receivers exposed to winds, electronic devices cooled by fans, heat exchanges placed in a low-velocity environment, etc. The mixed convection flows become important when the buoyancy forces due to the temperature difference between the wall and the free stream becomes large.

The study and analysis of heat and mass transfer in porous media has been the subject of many investigations due to their frequent occurrence in industrial and technological applications. Examples of some applications include geothermal reservoirs, drying of porous solids, thermal insulations and many others. Smith [1] initiated the study of the unsteady incompressible forced convection boundary-layer flow past a semi-infinite wedge impulsively set into motion. This problem subsequently solved numerically by Nanbu [2] using the method proposed by Hall [3] and then that modified by Harris et al. [4]. This method solves the untransformed equations directly using an iterative procedure and by implicit finite difference method, which is well documented and widely used by Keller and Cebeci [5] and also by Hossain [6] [7] and Hossain et al. [8] for unsteady boundary layers. Watkins [9] has solved this problem numerically following a second order; he has also studied the unsteady heat transfer aspects of the semi-infinite wedge started impulsively from rest to include solutions of the energy equation. A new set of scaled coordinates introduced by Williams and Rhyne [10].

There is a large body of literature on unsteady, mixed convection, boundarylayer flows past bodies of different geometries. Ibrahim et al. [11] studied the problem of unsteady magneto hydrodynamic (MHD) micropolar fluid flow and heat transfer over a vertical porous plate through a porous medium in the presence of thermal and mass diffusion with constant heat source. The unsteady free convection flow in the stagnation-point region of a rotating sphere embedded in a porous medium has analyzed by Hassanien et al. [12]. Ibrahim [13] has investigated the problem of unsteady mixed conviction flow in the stagnation region of a three dimensional body embedded in a porous medium. Al-Harbi and Hassanien [14] have studied the problem of unsteady mixed convection flow in the stagnation region of a heated vertical plate embedded in a variable porosity medium with thermal dispersion effects. Also, Al-Harbi [15] has analyzed the problem of unsteady mixed convection flow on a rotating cone embedded in a porous medium saturated with a rotating fluid. Unsteady magneto hydrodynamic mixed convection flow of an electrically conducting nanofluid in a stagnation region of a rotating sphere is studied numerically through Sameh and Rashed [16].

The interested reader can find an excellent collection of papers on unsteady convective flow problems over heated bodies embedded in a fluid-saturated porous medium in the book by Pop and Ingham [17] and in the book by Nield and Bejan [18]. 
Nanofluids with enhanced thermal characteristics have widely been examined to improve the heat transfer performance of many engineering applications [19]. Most researchers argue that the addition of nanoparticles with relatively higher thermal conductivity to the base fluid results in an increase of the thermal performance of the resultant nanofluid [20] [21] [22] [23].

According to Yacob et al. [24], nanofluids are produced by dispersing the nanometer-scale solid particles into base liquids with low thermal conductivity such as water and ethylene glycol. Nanoparticles are usually made of metal, metal oxide, carbide, nitride and even immiscible nano-scale liquid droplets. Gorla et al. [25] presented a boundary layer analysis for the mixed convection past a vertical wedge in a porous medium saturated with a nanofluid. Hamad et al. [26] introduced a one parameter group to represent similarity reductions for the problem of magnetic field effects on free-convective nanofluid flow past a semi-infinite vertical flat plate following a nanofluid model proposed by Buongiorno [27]. Hamad [28] obtained the analytical solutions for convective flow and heat transfer of a viscous incompressible nanofluid past a semi-infinite vertical stretching sheet in the presence of magnetic field. Khan and Pop [29] obtained similar solutions depending on Prandtl number, Lewis number, Brownian motion number and thermophoresis number on the steady boundary layer flow, heat and mass over a stretching surface in its plane. Further, Abu-Nada and Chamkha [30] have presented the natural convection heat transfer characteristics in a differentially heated enclosure filled with a CuO-EG-water nanofluid for different variable thermal conductivity and variable viscosity models. For more information, see also Das et al. [31], and Kakaç and Pramuanjaroenkij [32]. Muthtamilselvan et al. [33] claimed that it is difficult to have a precise idea on how nanoparticle enhances the heat transfer characteristics of nanofluids. More recently, Hady et al. [34] studied a nonsimilar solution of steady forced convection boundary layer flow and heat transfer of a nanofluid past a stretching horizontal late sing one-phase model. On the other hand, the same authors [35] extended the problem of Cheng-Minkowycz problem for natural convective boundary-layer flow in a porous medium saturated by a nanofluid to study the effect of the porosity of the plate. In addition, Hady et al. [36] studied the problem of the flow and heat transfer characteristics of a viscous nanofluid over a nonlinearly stretching sheet in the presence of thermal radiation and variable wall temperature. James et al. [37] have studied the effects of variable viscosity of nanofluid flow over a permeable wedge embedded in saturated non-Darcy porous medium with chemical reaction and thermal radiation. In existence of concerning magnetic field, heat together with mass transfer features on mixed convective copper-water nanofluid flow through inclined plate is investigated in surrounding porous medium together with viscous dissipation through Nasir et al. [38]. Chamkha et al. [39] introduced a mathematical model to accentuated the mixed bioconvective flow on a vertical wedge in a Darcy porous medium filled with a nanofluid containing both nanoparticles and gyrotactic microorganisms.

The aim of the present paper is to study the unsteady mixed convection flow 
along a symmetric wedge embedded in a porous medium saturated with a nanofluid in the presence of first and second orders resistances, which to the best of our knowledge have not been investigated yet. Motivation to study mixed convection in porous media comes from the need to characterize the convective transport processes around deep geological repository for the disposal of highlevel nuclear waste, e.g. spent fuel rods from nuclear reactors (see Lai [40]).

The effect of the presence of the buoyancy forces and the isotropic solid matrix on the unsteady mixed convection flow along a symmetric wedge embedded in a porous medium saturated with a nanofluid are considered. In addition, the Brownian motion and the thermophoresis effects are considered. The unsteadiness in the flow field is caused by impulsively creating motion in the free stream and at the same time suddenly raising the surface temperature above its surroundings. The partial differential equations governing the flow and the heat transfer solved numerically using some different numerical methods as the finite difference scheme by Pereyra [41]. Particular cases of the present results compared with those of Scshadri et al. [42].

\section{Governing Equations}

Let us consider an unsteady mixed convection boundary layer flow of an incompressible fluid past along a symmetric wedge with variable surface temperature embedded in a porous medium saturated with a nanofluid as shown in Figure 1 . The inviscid flow over the wedge develops instantaneously and its velocity is given by

$$
\bar{u}_{e}(\bar{x})=U_{\infty}\left(\frac{\bar{x}}{L}\right)^{m} \quad \text { for } m \leq 1
$$

where $L$ is a characteristic length and $m$ is pressure gradient related in the included angle $\pi \beta$ by $m=\beta /(2-\beta)$. It is clear that for negative values of $m$ the solution becomes singular at $\bar{x}=0$. While for $m$ positive the solution can be defined for all values of $\bar{x}$. It is assumed that the variable surface temperature of the wedge is $\left(T_{w}>T_{\infty}\right)$ where $T_{\infty}$ is the ambient temperature of the fluid and

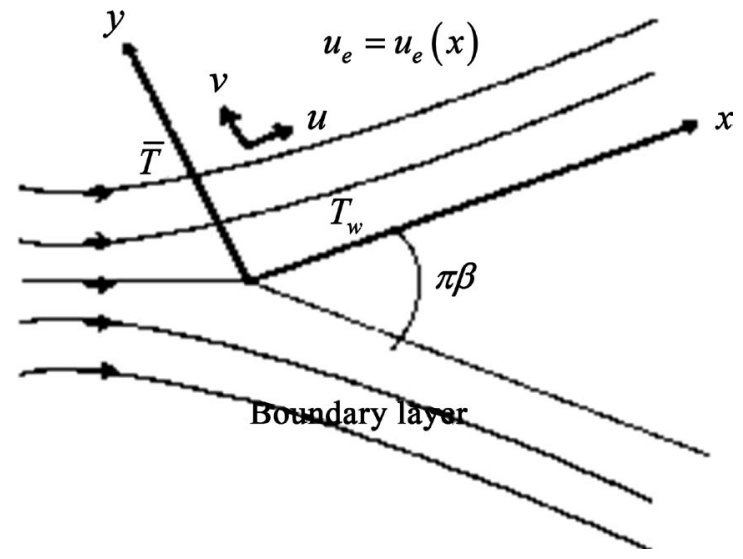

Figure 1. Physical model and coordinate system. 
$\bar{T}$ is the temperature of the fluid. Under the above-mentioned assumptions, the boundary layer equations governing the flow can be expressed as follows:-

$$
\begin{gathered}
\frac{\partial \bar{u}}{\partial \bar{x}}+\frac{\partial \bar{v}}{\partial \bar{y}}=0 \\
\frac{\partial \bar{u}}{\partial \bar{t}}+\bar{u} \frac{\partial \bar{u}}{\partial \bar{x}}+\bar{v} \frac{\partial \bar{u}}{\partial \bar{y}} \\
=\bar{u}_{e} \frac{\mathrm{d} \bar{u}_{e}}{\mathrm{~d} \bar{x}}+v \frac{\partial^{2} \bar{u}}{\partial \bar{y}^{2}}+\frac{v \varepsilon}{K}\left(\bar{u}_{e}-\bar{u}\right)+\frac{\Gamma \varepsilon^{2}}{K^{1 / 2}}\left(\bar{u}_{e}^{2}-\bar{u}^{2}\right) \\
+\left[\left(1-\varphi_{\infty}\right) \beta_{T} \rho_{f}\left(\bar{T}-T_{\infty}\right)-\left(\rho_{p}-\rho_{f}\right)\left(\bar{\varphi}-\varphi_{\infty}\right) / \rho_{f}\right] g \\
\frac{\partial \bar{T}}{\partial \bar{t}}+\bar{u} \frac{\partial \bar{T}}{\partial \bar{x}}+\bar{v} \frac{\partial \bar{T}}{\partial \bar{y}}=\alpha_{m} \frac{\partial^{2} \bar{T}}{\partial \bar{y}^{2}}+\tau\left[D_{B} \frac{\partial \bar{T}}{\partial \bar{y}} \frac{\partial \bar{\varphi}}{\partial \bar{y}}+\frac{D_{T}}{T_{\infty}}\left(\frac{\partial \bar{T}}{\partial y}\right)^{2}\right], \\
\quad \frac{\partial \bar{\varphi}}{\partial \bar{t}}+\bar{u} \frac{\partial \bar{\varphi}}{\partial \bar{x}}+\bar{v} \frac{\partial \bar{\varphi}}{\partial \bar{y}}=D_{B} \frac{\partial^{2} \bar{\varphi}}{\partial \bar{y}^{2}}+\frac{D_{T}}{T_{\infty}}\left(\frac{\partial^{2} \bar{T}}{\partial \bar{y}^{2}}\right),
\end{gathered}
$$

where $\bar{u}$ and $\bar{v}$ are the velocity components in is $\bar{x}$ and $\bar{y}$ coordinate, respectively. $\bar{T}$ is the temperature, $\bar{\varphi}$ is the nanoparticle volume fraction, $g$ is the acceleration due to gravity, $\rho_{f}$ is the density of the base fluid and $v$ and $\beta_{T}$ are the viscosity and thermal conductivity of the fluid. While, $\rho_{p}$ is the density of the nanoparticles, $(\rho c)_{f}$ is the heat capacity of the fluid and $(\rho c)_{p}$ is the effective heat capacity of the nanoparticle material. $\alpha_{m}=k_{m} /(\rho c)_{f}$ is the thermal diffusivity, $\tau=(\rho c)_{p} /(\rho c)_{f}$ is the ratio of the effective heat capacity of the nanoparticle material to the heat capacity of the fluid and $k_{m}$ is the effective thermal conductivity of the porous medium. $K, \varepsilon$ and $\Gamma$ are the permeability, the porosity of the porous medium and the empirical constant in the secondorder resistance. The coefficients that appear in Equations ((4), (5)) are the Brownian diffusion coefficient $D_{B}$, and the thermophoretic diffusion coefficient $D_{T}$.

The boundary conditions for the present problem are

$$
\begin{aligned}
& \bar{u}=0, \quad \bar{v}=0, \quad \bar{T}=T_{w}(\bar{x})=T_{\infty}+\left(T_{r}-T_{\infty}\right)\left(\frac{\bar{x}}{L}\right)^{2 m-1}, \\
& \bar{\varphi}=\varphi_{w}(\bar{x})=\varphi_{\infty}+\left(\varphi_{r}-\varphi_{\infty}\right)\left(\frac{\bar{x}}{L}\right)^{2 m-1}, \text { at } \bar{y}=0, \\
& \bar{u}=\bar{u}_{e}(\bar{x}), \quad \bar{T} \rightarrow T_{\infty}, \quad \bar{\varphi} \rightarrow \varphi_{\infty} \quad \text { as } \bar{y} \rightarrow \infty .
\end{aligned}
$$

We introduce non dimensional dependent and independent variables according to,

$$
\begin{aligned}
& x=\frac{\bar{x}}{L}, \quad y=\operatorname{Re}_{L}^{1 / 2} \frac{\bar{y}}{L}, \quad u=\frac{\bar{u}}{U_{\infty}}, \quad v=R e_{L}^{1 / 2} \frac{\bar{v}}{U_{\infty}}, \quad u_{e}=\frac{\bar{u}_{e}}{U_{\infty}}, \\
& t=\frac{U_{\infty}}{L} \bar{t}, \quad T=\frac{\bar{T}-T_{\infty}}{T_{r}-T_{\infty}}, \quad \varphi=\frac{\bar{\varphi}-\varphi_{\infty}}{\varphi_{r}-\varphi_{\infty}} .
\end{aligned}
$$

The velocity over the wedge is now given by $u_{e}(x)=x^{m}$ for $m \leq 1$.

The governing Equations (2)-(5) can be written as 


$$
\begin{gathered}
\frac{\partial u}{\partial x}+\frac{\partial v}{\partial y}=0 \\
\frac{\partial u}{\partial t}+u \frac{\partial u}{\partial x}+v \frac{\partial v}{\partial y} \\
=u_{e} \frac{\mathrm{d} u_{e}}{\mathrm{~d} x}+\frac{\partial^{2} u}{\partial y^{2}}+\frac{\varepsilon}{D a_{L} R e_{L}}\left(u_{e}-u\right)+\frac{\Gamma \varepsilon^{2}}{D a_{L}^{1 / 2}}\left(u_{e}^{2}-u^{2}\right)+\lambda\left(T-N_{r} \varphi\right), \\
\frac{\partial T}{\partial t}+u \frac{\partial T}{\partial x}+v \frac{\partial T}{\partial y}=\frac{1}{P r} \frac{\partial^{2} T}{\partial y^{2}}+N_{o B} \frac{\partial T}{\partial y} \frac{\partial \varphi}{\partial y}+N_{o T}\left(\frac{\partial T}{\partial y}\right)^{2}, \\
\frac{\partial \varphi}{\partial t}+u \frac{\partial \varphi}{\partial x}+v \frac{\partial \varphi}{\partial y}=\frac{1}{S c} \frac{\partial^{2} \varphi}{\partial y^{2}}+\frac{1}{S c} \frac{N_{o T}}{N_{o B}} \frac{\partial^{2} T}{\partial y^{2}}
\end{gathered}
$$

where $D a_{L}=K / L^{2}$ is the Darcy number, $\lambda=G r_{L} / R e_{L}^{2}$ is the mixed convection parameter, $R e_{L}=U_{\infty} L / v$ is the Reynolds number, $G r_{L}=g \beta\left(T_{r}-T_{\infty}\right) L^{3} / v^{2}$ is the Grashof number, $N_{r}=\frac{\left(\rho_{p}-\rho_{f}\right)\left(\varphi_{r}-\varphi_{\infty}\right)}{\rho_{f}\left(1-\varphi_{\infty}\right)\left(T_{r}-T_{\infty}\right)}$ is the nanofluid buoyancy ratio parameter, $N_{o T}=\frac{\tau}{v}\left(\frac{D_{T}}{T_{\infty}}\right)\left(T_{r}-T_{\infty}\right)$ is the thermophores is parameter, $N_{o B}=\frac{\tau}{v} D_{B}\left(\varphi_{r}-\varphi_{\infty}\right)$ is the Brownian motion parameter, $\operatorname{Pr}=\alpha_{m} / v$ is the Prandtl number and $S c=v / B_{B}$ is the Schmidt number.

For $t \geq 0$ and $m \leq 1$ the boundary conditions then may be written as:

$$
\begin{aligned}
& u=0, \quad v=0, \quad T=x^{2 m-1}, \varphi=x^{2 m-1} \text { at } y=0, \\
& u=u_{e}(x)=x^{m}, \quad T \rightarrow 0, \varphi \rightarrow 0 \text { as } y \rightarrow \infty
\end{aligned}
$$

The number of independent variables in the governing Equations (8)-(11) can be reduced from three to two by applying the following transformations as

$$
\begin{aligned}
& \eta=x^{(m-1) / 2}(2 \xi)^{-1 / 2} y, \quad \psi=x^{(m+1) / 2}(2 \xi)^{1 / 2} f(\eta, \xi), \\
& T=x^{2 m-1} \theta(\eta, \xi), \varphi=x^{2 m-1} \phi(\eta, \xi), \quad \xi=1-\mathrm{e}^{-\tau}, \tau=x^{m-1} t,
\end{aligned}
$$

where $\eta$ is a non-dimensional similarity variable and $\psi$ is the stream function, which is defined in the usual way, namely $u=\partial \psi / \partial y$ and $v=-\partial \psi / \partial y$.

Substituting the transformations (13) into Equations (9)-(11), we obtained the following transformed equations for the momentum and thermal boundary layer equations:

$$
\begin{aligned}
& f^{\prime \prime \prime}+\eta(1-\xi) f^{\prime \prime}+[(m+1) \xi-(m-1)(1-\xi) \ln (1-\xi)] f f^{\prime \prime} \\
& +2 \xi\left[\gamma_{x}\left(1-f^{\prime}\right)+\left(m+\Delta_{x}\right)\left(1-f^{\prime 2}\right)\right] \\
& =2(1-m) \xi(1-\xi) \ln (1-\xi)\left(f^{\prime} \frac{\partial f^{\prime}}{\partial \xi}-f^{\prime \prime} \frac{\partial f}{\partial \xi}\right) \\
& +2 \xi(1-\xi) \frac{\partial f^{\prime}}{\partial \xi}-2 \lambda \xi\left(\theta-N_{r} \phi\right),
\end{aligned}
$$




$$
\begin{aligned}
& \frac{1}{P r} \theta^{\prime \prime}+\eta(1-\xi) \theta^{\prime}+[(m+1) \xi-(m-1)(1-\xi) \ln (1-\xi)] f \theta^{\prime} \\
&-2(2 m-1) \xi \theta f^{\prime} \\
&= 2(1-m) \xi(1-\xi) \ln (1-\xi)\left(f^{\prime} \frac{\partial \theta}{\partial \xi}-\theta^{\prime} \frac{\partial f}{\partial \xi}\right) \\
&+2 \xi(1-\xi) \frac{\partial \theta}{\partial \xi}-N_{B} \theta^{\prime} \phi^{\prime}-N_{T} \theta^{\prime 2} \\
& \frac{1}{S c} \phi^{\prime \prime}+\eta(1-\xi) \phi^{\prime}+[(m+1) \xi-(m-1)(1-\xi) \ln (1-\xi)] f \phi^{\prime} \\
&-2(2 m-1) \xi \phi f^{\prime} \\
&=2(1-m) \xi(1-\xi) \ln (1-\xi)\left(f^{\prime} \frac{\partial \phi}{\partial \xi}-\phi^{\prime} \frac{\partial f}{\partial \xi}\right)+2 \xi(1-\xi) \frac{\partial \phi}{\partial \xi}-\frac{N_{T}}{S c N_{B}} \theta^{\prime \prime}
\end{aligned}
$$

where $\gamma_{x}=D a^{-1} R e_{L}^{-1} x^{1-m}$ is the local permeability parameter, $\Delta_{x}=F x$ is the inertia coefficient parameter (see Chamkha [23]). The modified thermophoresis and Brownian motion parameters are defined as:

$$
\begin{aligned}
& N_{T}=N_{o T} x^{2 m-1}=\frac{\tau}{v}\left(\frac{D_{T}}{T_{\infty}}\right)\left(T_{r}-T_{\infty}\right) x^{2 m-1}=\frac{\tau}{v}\left(\frac{D_{T}}{T_{\infty}}\right)\left(T_{w}-T_{\infty}\right), \\
& N_{B}=N_{o B} x^{2 m-1}=\frac{\tau}{v} D_{B}\left(\varphi_{r}-\varphi_{\infty}\right) x^{2 m-1}=\frac{\tau}{v} D_{B}\left(\varphi_{w}-\varphi_{\infty}\right)
\end{aligned}
$$

The boundary conditions to be satisfied by the Equations (14)-(16) are

$$
\begin{aligned}
& f(0, \xi)=f^{\prime}(0, \xi)=0, \quad \theta(0, \xi)=1, \quad \phi(0, \xi)=1, \\
& f^{\prime}(\infty, \xi)=1, \quad \theta(\infty, \xi)=0, \quad \phi(\infty, \xi)=0,
\end{aligned}
$$

In the above equations, prime denotes differentiation of the functions with respect to $\eta$ only.

Now to find the numerical solutions we can get the easiest form by using the transformations $\xi=1-\mathrm{e}^{-\tau}$ from Equations (14)-(16) applicable for $0 \leq \tau \leq \infty$.

$$
\begin{gathered}
f^{\prime \prime \prime}+\eta \mathrm{e}^{-\tau} f^{\prime \prime}+\left[(m+1)\left(1-\mathrm{e}^{-\tau}\right)+(m-1) \tau \mathrm{e}^{-\tau}\right] f f^{\prime \prime} \\
+2\left(1-\mathrm{e}^{-\tau}\right)\left[\gamma_{x}\left(1-f^{\prime}\right)+\left(m+\Delta_{x}\right)\left(1-f^{\prime 2}\right)\right] \\
=2(m-1) \tau\left(1-\mathrm{e}^{-\tau}\right)\left(f^{\prime} \frac{\partial f^{\prime}}{\partial \tau}-f^{\prime \prime} \frac{\partial f}{\partial \tau}\right)+2\left(1-\mathrm{e}^{-\tau}\right)\left(\frac{\partial f^{\prime}}{\partial \tau}\right) \\
-2 \lambda\left(1-\mathrm{e}^{-\tau}\right)\left(\theta-N_{r} \phi\right) \\
\frac{1}{P r} \theta^{\prime \prime}+\eta \mathrm{e}^{-\tau} \theta^{\prime}+\left[(m+1)\left(1-\mathrm{e}^{-\tau}\right)+(m-1) \tau \mathrm{e}^{-\tau}\right] f \theta^{\prime} \\
-2(2 m-1)\left(1-\mathrm{e}^{-\tau}\right) \theta f^{\prime} \\
=2(m-1) \tau\left(1-\mathrm{e}^{-\tau}\right)\left(f^{\prime} \frac{\partial \theta}{\partial \tau}-\theta^{\prime} \frac{\partial f}{\partial \tau}\right)+2\left(1-\mathrm{e}^{-\tau}\right) \frac{\partial \theta}{\partial \tau}-N_{B} \theta^{\prime} \phi^{\prime}-N_{T} \theta^{\prime 2} \\
\frac{1}{S c} \phi^{\prime \prime}+\eta \mathrm{e}^{-\tau} \phi^{\prime}+\left[(m+1)\left(1-\mathrm{e}^{-\tau}\right)+(m-1) \tau \mathrm{e}^{-\tau}\right] f \phi^{\prime}-2(2 m-1)\left(1-\mathrm{e}^{-\tau}\right) \phi f^{\prime} \\
=2(m-1) \tau\left(1-\mathrm{e}^{-\tau}\right)\left(f^{\prime} \frac{\partial \phi}{\partial \tau}-\phi^{\prime} \frac{\partial f}{\partial \tau}\right)+2\left(1-\mathrm{e}^{-\tau}\right) \frac{\partial \phi}{\partial \tau}-\frac{N_{T}}{S c N_{B}} \theta^{\prime \prime}
\end{gathered}
$$


The boundary conditions to be satisfied by the above equations are

$$
\begin{aligned}
& f(0, \tau)=f^{\prime}(0, \tau)=0, \theta(0, \tau)=1, \phi(0, \tau)=1, \\
& f^{\prime}(\infty, \tau)=1, \theta(\infty, \tau)=0, \quad \theta(\infty, \tau)=0 .
\end{aligned}
$$

In practical applications, two quantities of physical interest are to be determined, such as, surface shear stress and the rate of heat and mass transfer at the surface. These may obtained in terms of the skin friction coefficient (wall shear stress) $C_{f}$, local Nusselt number $N u_{x}$ and the local Sherwood number $S h_{x}$, which are defined by:

$$
\begin{aligned}
C_{f} & =\frac{\mu}{\rho_{f} U_{e}^{2}(\bar{x})}\left(\frac{\partial \bar{u}}{\partial \bar{y}}\right)_{y=0}, N u_{x}=\frac{\bar{x}}{T_{w}-T_{\infty}}\left(\frac{\partial \bar{T}}{\partial \bar{y}}\right)_{y=0}, \\
S h_{x} & =\frac{\bar{x}}{\varphi_{w}-\varphi_{\infty}}\left(\frac{\partial \bar{\varphi}}{\partial \bar{y}}\right)_{y=0}
\end{aligned}
$$

By introducing the non-dimensional variables (7) and the transformation (13), the skin friction coefficient, $C_{f}$, the local Nusselt number, $N u_{x}$ and the local Sherwood number, $S h_{x}$ can now defined by:

$$
\begin{aligned}
& C_{f} R e_{x}^{1 / 2}=\left[2\left(1-\mathrm{e}^{-\tau}\right)\right]^{-1 / 2} f^{\prime \prime}(0, \tau), \\
& N u_{x} R e_{x}^{-1 / 2}=-\left[2\left(1-\mathrm{e}^{-\tau}\right)\right]^{-1 / 2} \theta^{\prime}(0, \tau), \\
& S h_{x} R e_{x}^{-1 / 2}=-\left[2\left(1-\mathrm{e}^{-\tau}\right)\right]^{-1 / 2} \phi^{\prime}(0, \tau) .
\end{aligned}
$$

Perturbation solutions method for small time $\tau \ll 1$

For small $\tau$ Equations (19)-(21) become

$$
\begin{gathered}
f^{\prime \prime \prime}+\eta f^{\prime \prime}+2 m \tau f f^{\prime \prime}+2 \tau\left[\gamma_{x}\left(1-f^{\prime}\right)+\left(m+\Delta_{x}\right)\left(1-f^{\prime 2}\right)+\lambda\left(\theta-N_{r} \phi\right)\right] \\
=2(m-1) \tau^{2}\left[f^{\prime} \frac{\partial f^{\prime}}{\partial \tau}-f^{\prime \prime} \frac{\partial f}{\partial \tau}\right]+2 \tau\left[\frac{\partial f^{\prime}}{\partial \tau}\right] \\
\frac{1}{P r} \theta^{\prime \prime}+\eta \theta^{\prime}+2 m \tau f \theta^{\prime}-2(2 m-1) \tau \theta f^{\prime}+N_{B} \theta^{\prime} \phi^{\prime}+N_{T} \theta^{\prime 2} \\
=2(1-m) \tau^{2}\left[f^{\prime} \frac{\partial \theta}{\partial \tau}-\theta^{\prime} \frac{\partial f}{\partial \tau}\right]+2 \tau \frac{\partial \theta}{\partial \tau} \\
\frac{1}{S c} \phi^{\prime \prime}+\eta \phi^{\prime}+2 m \tau f \phi^{\prime}-2(2 m-1) \tau \phi f^{\prime}+\frac{N_{T}}{S c N_{B}} \theta^{\prime \prime} \\
=2(1-m) \tau^{2}\left[f^{\prime} \frac{\partial \phi}{\partial \tau}-\phi^{\prime} \frac{\partial f}{\partial \tau}\right]+2 \tau \frac{\partial \phi}{\partial \tau}
\end{gathered}
$$

and the corresponding boundary conditions (22) become

$$
\begin{aligned}
& f(0, \tau)=f^{\prime}(0, \tau)=0, \theta(0, \tau)=1, \phi(0, \tau)=1, \\
& f^{\prime}(\infty, \tau)=1, \theta(\infty, \tau)=0, \phi(\infty, \tau)=0
\end{aligned}
$$

The resulting system of Equations (25)-(27), along with boundary conditions (28) is solved using the perturbation technique. Now, the non-dimensional stream function and the temperature functions can be written as: 


$$
\begin{aligned}
& f(\eta, \tau)=f_{0}(\eta)+\tau f_{1}(\eta)+\tau^{2} f_{2}(\eta)+\cdots \\
& \theta(\eta, \tau)=\theta_{0}(\eta)+\tau \theta_{1}(\eta)+\tau^{2} \theta_{2}(\eta)+\cdots \\
& \phi(\eta, \tau)=\phi_{0}(\eta)+\tau \phi_{1}(\eta)+\tau^{2} \phi_{2}(\eta)+\cdots
\end{aligned}
$$

Substituting Equations (29)-(31) into Equations (25)-(27) and equating the various coefficients of power of $\tau$ to zero (here we collect terms up to the second power of $O\left(\tau^{2}\right)$, we can get the following sets of ordinary differential equations:

Zero order:

$$
\begin{gathered}
f_{0}^{\prime \prime \prime}+\eta f_{0}^{\prime \prime}=0 \\
\frac{1}{P r} \theta_{0}^{\prime \prime}+\eta \theta_{0}^{\prime}+N_{B} \theta_{0}^{\prime} \phi_{0}^{\prime}+N_{T} \theta_{0}^{22}=0, \\
\frac{1}{S c} \phi_{0}^{\prime \prime}+\eta \phi_{0}^{\prime}+\frac{N_{T}}{S c N_{B}} \theta_{0}^{\prime \prime}=0,
\end{gathered}
$$

with the corresponding boundary conditions:

$$
f_{0}(0)=f_{0}^{\prime}(0)=0, \quad \theta_{0}(0)=\phi_{0}(0)=1, \quad f_{0}^{\prime}(\infty)=1, \quad \theta_{0}(\infty)=\phi_{0}(\infty)=0
$$

\section{First order:}

$$
\begin{gathered}
f_{1}^{\prime \prime \prime}+\eta f_{1}^{\prime \prime}-2 f_{1}^{\prime}=-2 m\left(1-f_{0}^{\prime 2}+f_{0} f_{0}^{\prime \prime}\right)+2\left(\gamma_{x} f_{0}^{\prime}+\Delta_{x} f_{0}^{\prime \prime 2}\right)-2 \lambda\left(\theta_{0}-N_{r} \phi_{0}\right), \\
\frac{1}{P r} \theta_{1}^{\prime \prime}+\eta \theta_{1}^{\prime}-2 \theta_{1}+N_{B} \theta_{1}^{\prime} \phi_{1}^{\prime}+N_{T} \theta_{1}^{\prime 2}=-2 m f_{0} \theta_{0}^{\prime}+2(2 m-1) \theta_{0} f_{0}^{\prime}, \\
\frac{1}{S c} \phi_{1}^{\prime \prime}+\eta \phi_{1}^{\prime}-2 \phi_{1}+\frac{N_{T}}{S c N_{B}} \theta_{1}^{\prime \prime}=-2 m f_{0} \phi_{0}^{\prime}+2(2 m-1) \phi_{0} f_{0}^{\prime},
\end{gathered}
$$

with the corresponding boundary conditions:

$$
f_{1}(0)=f_{1}^{\prime}(0)=0, \theta_{1}(0)=\phi_{1}(0)=0, \quad f_{1}^{\prime}(\infty)=0, \quad \theta_{1}(\infty)=\phi_{1}(\infty)=0
$$

\section{Second order:}

$$
\begin{aligned}
& f_{2}^{\prime \prime \prime}+\eta f_{2}^{\prime \prime}-4 f_{2}^{\prime} \\
& =2\left[(1-2 m) f_{1} f_{0}^{\prime \prime}+(3 m-1) f_{1}^{\prime} f_{0}^{\prime}-m f_{0} f_{1}^{\prime \prime}-\lambda\left(\theta_{1}-N_{r} \phi_{1}\right)\right]+2 \gamma_{x} f_{1}^{\prime}+4 \Delta_{x} f_{0}^{\prime} f_{1}^{\prime} \\
& \quad \frac{1}{P r} \theta_{2}^{\prime \prime}+\eta \theta_{2}^{\prime}-4 \theta_{2}+N_{B} \theta_{2}^{\prime} \phi_{2}^{\prime}+N_{T} \theta_{2}^{\prime 2} \\
& =2\left[(1-2 m) f_{1} \theta_{0}^{\prime}+(3 m-2) \theta_{1} f_{0}^{\prime}-m \theta_{1}^{\prime} f_{0}+(2 m-1) \theta_{0} f_{1}^{\prime}\right] \\
& \frac{1}{S c} \phi_{2}^{\prime \prime}+\eta \phi_{2}^{\prime}-4 \phi_{2}+\frac{N_{T}}{S c N_{B}} \theta_{2}^{\prime \prime} \\
& =2\left[(1-2 m) f_{1} \phi_{0}^{\prime}+(3 m-2) \phi_{1} f_{0}^{\prime}-m \phi_{1}^{\prime} f_{0}+(2 m-1) \phi_{0} f_{1}^{\prime}\right]
\end{aligned}
$$

With the corresponding boundary conditions:

$$
f_{2}(0)=f_{2}^{\prime}(0)=0, \theta_{2}(0)=0, \theta_{2}(0)=0, f_{2}^{\prime}(\infty)=0, \theta_{2}(\infty)=0, \phi_{2}(\infty)=0
$$

where primes denote differentiation with respect to $\eta$. Knowing the values of $f_{0}^{\prime \prime}(0), f_{1}^{\prime \prime}(0), f_{2}^{\prime \prime}(0), \theta_{0}^{\prime}(0), \theta_{1}^{\prime}(0), \theta_{2}^{\prime}(0), \phi_{0}^{\prime}(0), \phi_{1}^{\prime}(0)$ and $\phi_{2}^{\prime}(0)$ from the solutions of Equations (32)-(43), we get the values of the skin friction coefficient $C_{f}$, local Nusselt number $N u_{x}$ and the local Sherwood number $S h_{x}$, from the fol- 
lowing expressions:

$$
\begin{aligned}
& C_{f} R e_{x}^{1 / 2}=(2 \tau)^{-1 / 2}\left(f_{0}^{\prime \prime}(0)+\tau f_{1}^{\prime \prime}(0)+\tau^{2} f_{2}^{\prime \prime}(0)\right), \\
& N u_{x} R e_{x}^{-1 / 2}=-(2 \tau)^{-1 / 2}\left(\theta_{0}^{\prime}(0)+\tau \theta_{1}^{\prime}(0)+\tau^{2} \theta_{2}^{\prime}(0)\right), \\
& S h_{x} \operatorname{Re}_{x}^{-1 / 2}=(2 \tau)^{-1 / 2}\left(\phi_{0}^{\prime}(0)+\tau \phi_{1}^{\prime}(0)+\tau^{2} \phi_{2}^{\prime}(0)\right) .
\end{aligned}
$$

\section{Results and Discussion}

The sets of ordinary differential Equations (32)-(34) are solved successively by giving appropriate initial guess values for $f_{i}^{\prime \prime}(0), \theta_{i}^{\prime}(0), \phi_{i}^{\prime}(0), i=0,1,2$ to match the values with the corresponding boundary conditions at

$f_{i}^{\prime}(\infty), \theta_{i}(\infty), \phi_{i}(\infty), i=0,1,2$. The numerical values of the coefficients of skin friction, $C_{f} R e_{x}^{1 / 2}$ and the Nusselt number $N u_{x} R e_{x}^{-1 / 2}$ for smaller values of $\tau \in[0,1]$ obtained by perturbation method while $P r=1.0$ and $m=0.2$ are shown in Table 1 in the absence of the effect of the buoyancy force $(\lambda=0)$, thermophoresis parameter $\left(N_{T}=0\right)$ and Brownian motion parameter $\left(N_{B}=0\right)$. These values compared with that of Hossain et al. [8] and Harris et al. [4]. The comparison shows excellent agreement between these two results.

Numerical results presented for some representative values of the governing parameters govern this problem. In order to see the physical insight, the numerical values of velocity $f^{\prime}(\eta)$, temperature $\theta(\eta)$, and nanoparticle volume fraction $\phi(\eta)$ within the boundary layer computed for different parameters as unsteadiness parameter $\tau$, mixed convection parameter $\lambda$, nanofluid buoyancy ratio parameter $N_{t}$, thermophoresis parameter $N_{T}$, Brownian motion parameter $N_{B}$, first resistant parameter $\gamma$, second resistant parameter $\Delta$, Prandtl number $\operatorname{Pr}$ and Schmidt number $S c$. In addition, numerical results obtained to discuss the effects of the governing parameters on the skin friction coefficient, local Nussel number and local Shrewood number and displayed in tabular and graphical forms.

Figures 2-4 displayed the effects of nanonfluid parameters, thermophoresis parameter $N_{T}$, Brownian motion parameter $N_{B}$ and buoyancy ratio parameter $N_{r}$ From these figures, it is observed that the velocity and the temperature profiles

Table 1. Comparison of the present numerical results of $C_{f} R e_{x}^{1 / 2}$ and $N u_{x} R e_{x}^{-1 / 2}$ for different values of small time while $P r=1.0$ and $m=0.2$.

\begin{tabular}{ccccccc}
\hline \multirow{2}{*}{$\tau$} & \multicolumn{2}{c}{ Harris et al. [4] } & \multicolumn{2}{c}{ Hossian $e t$ al. [11] } & \multicolumn{2}{c}{ Present result } \\
\cline { 2 - 7 } & $C_{f} R e_{x}^{1 / 2}$ & $N u_{x} R e_{x}^{-1 / 2}$ & $C_{f} R e_{x}^{1 / 2}$ & $N u_{x} R e_{x}^{-1 / 2}$ & $C_{f} R e_{x}^{1 / 2}$ & $N u_{x} R e_{x}^{-1 / 2}$ \\
\hline 0.01 & 5.65797 & 5.64360 & 5.66886 & 5.64525 & 5.65769 & 5.63468 \\
0.10 & 1.83491 & 1.78946 & 1.83693 & 1.76140 & 1.83312 & 1.75951 \\
0.20 & 1.33334 & 1.26902 & 1.33189 & 1.22357 & 1.33090 & 1.22543 \\
0.40 & 0.99341 & 0.90234 & 0.99548 & 0.83937 & 0.99038 & 0.83739 \\
1.0 & 0.72370 & 0.57925 & 0.72665 & 0.46370 & 0.72116 & 0.46104 \\
\hline
\end{tabular}




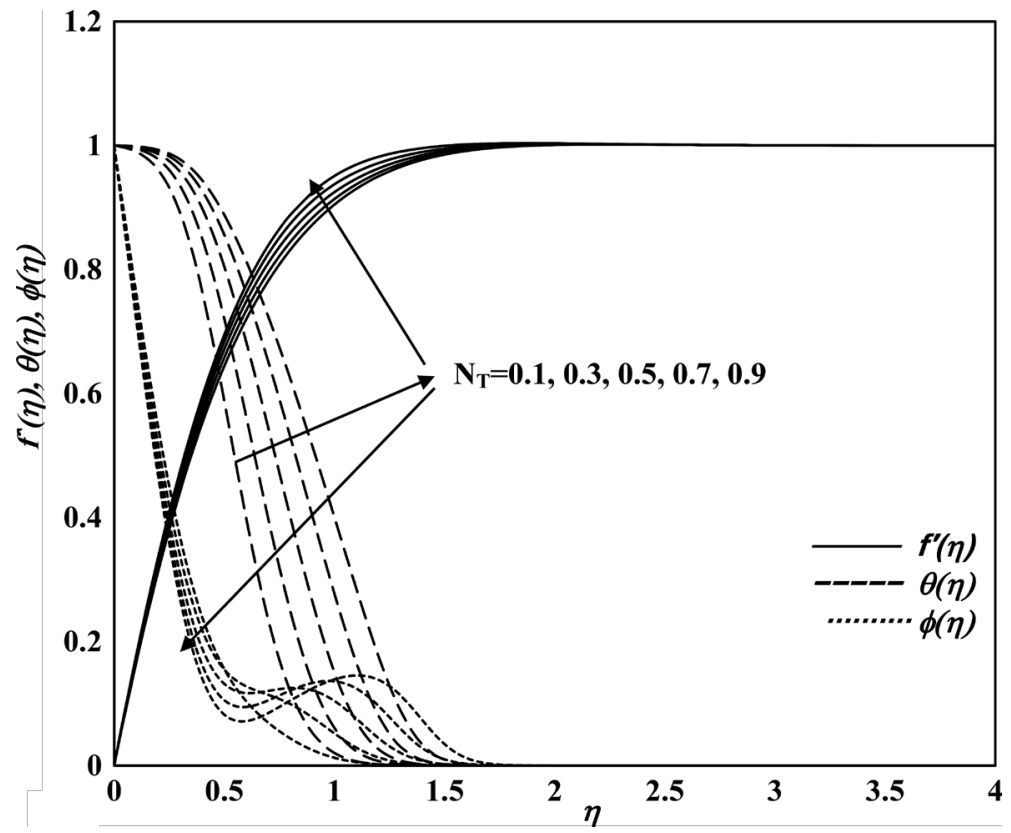

Figure 2. Effect of thermophoresis parameter $N_{T}$ on velocity $f^{\prime}(\eta)$, temperature $\theta(\eta)$ and nanoparticle volume fraction $\phi(\eta)$ with $N_{r}=N_{B}=m=\gamma=\Delta=0.5, \lambda=1$, $\operatorname{Pr}=S c=10$ and $\tau=0.5$

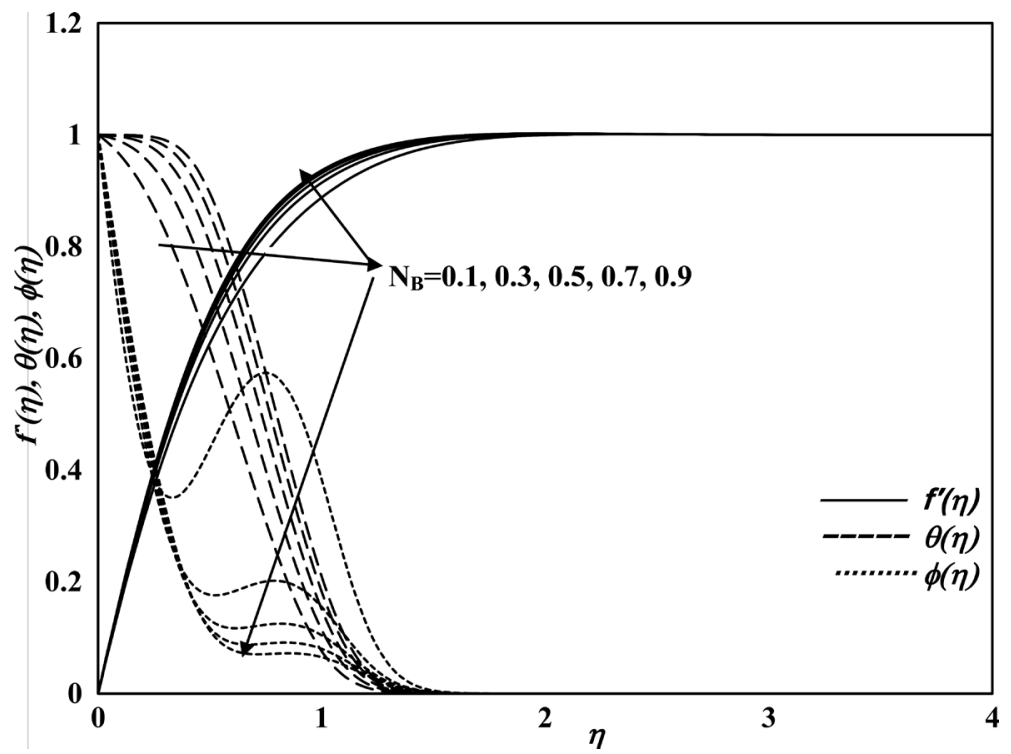

Figure 3. Effect of Brownian motion parameter $N_{B}$ on velocity $f^{\prime}(\eta)$, temperature $\theta(\eta)$ and nanoparticle volume fraction $\phi(\eta)$ with $N_{r}=N_{T}=m=\gamma=\Delta=0.5, \lambda=1, \operatorname{Pr}=S c=10$ and $\tau=0.5$.

increase with an increase in both $N_{T}$ and $N_{B}$. However, for the nanoparticle volume fraction profiles there is a crossing over point where the volume fraction profile decreases before that point and slightly increases after that (Figure 2 and Figure 3). Hence, the heat transfer enhancement is due to collision of high thermal energy with lower energy particles. From Figure 4, it is observed that 
with the increase in the buoyancy ratio parameter $N_{r}$, the velocity profile decreases and both the temperature and volume fraction profiles slightly increase.

The effects of the mixed convection parameter $\lambda$ on the non-dimensional velocity, temperature and nanoparticle volume fraction are illustrated in Figure 5.

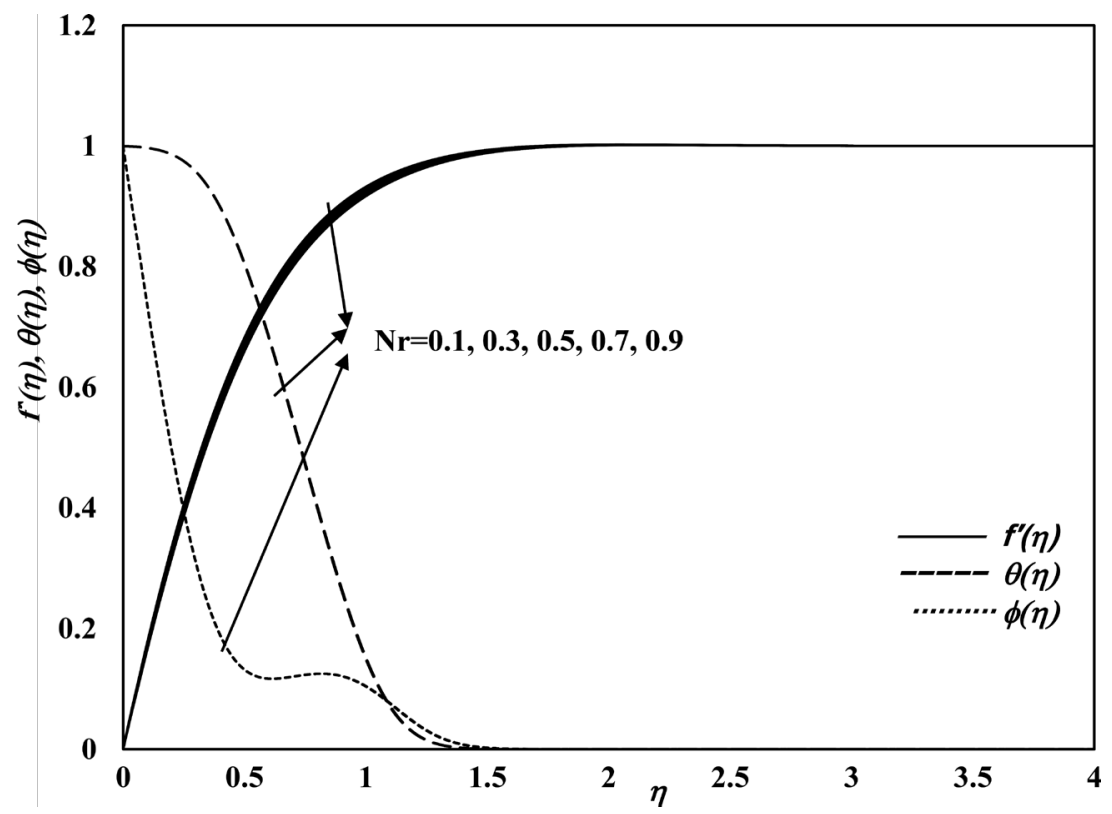

Figure 4. Effect of buoyancy ratio parameter $N_{r}$ on velocity $f^{\prime}(\eta)$, temperature $\theta(\eta)$ and nanoparticle volume fraction $\phi(\eta)$ with $N_{T}=N_{B}=m=\gamma=\Delta=0.5, \lambda=1, \operatorname{Pr}=S c=10$ and $\tau=0.5$.

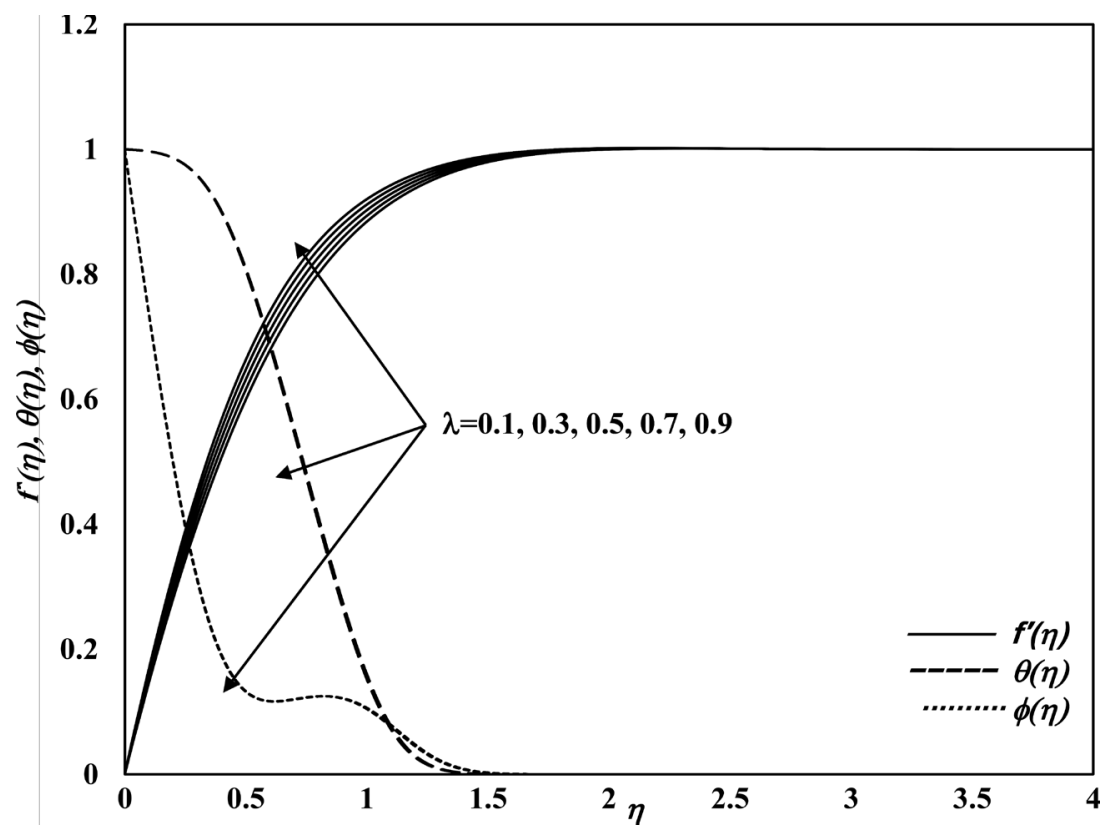

Figure 5. Effect of mixed convection parameter $\lambda$ on velocity $f^{\prime}(\eta)$, temperature $\theta(\eta)$ and nanoparticle volume fraction $\phi(\eta)$ with $N_{T}=N_{B}=N_{r}=m=\gamma=\Delta=0.5, \operatorname{Pr}=S c=10$ and $\tau=0.5$. 
From this figure, it is observed that the velocity increases as $\lambda$ increases, however; both the temperature and nanoparticle fraction profiles slightly decrease with increasing values of $\lambda$.

Figure 6 \& Figure 7 exhibit the effects of first resistant parameter $\gamma$ and second resistant parameter $\Delta$ of the porous medium on velocity, temperature

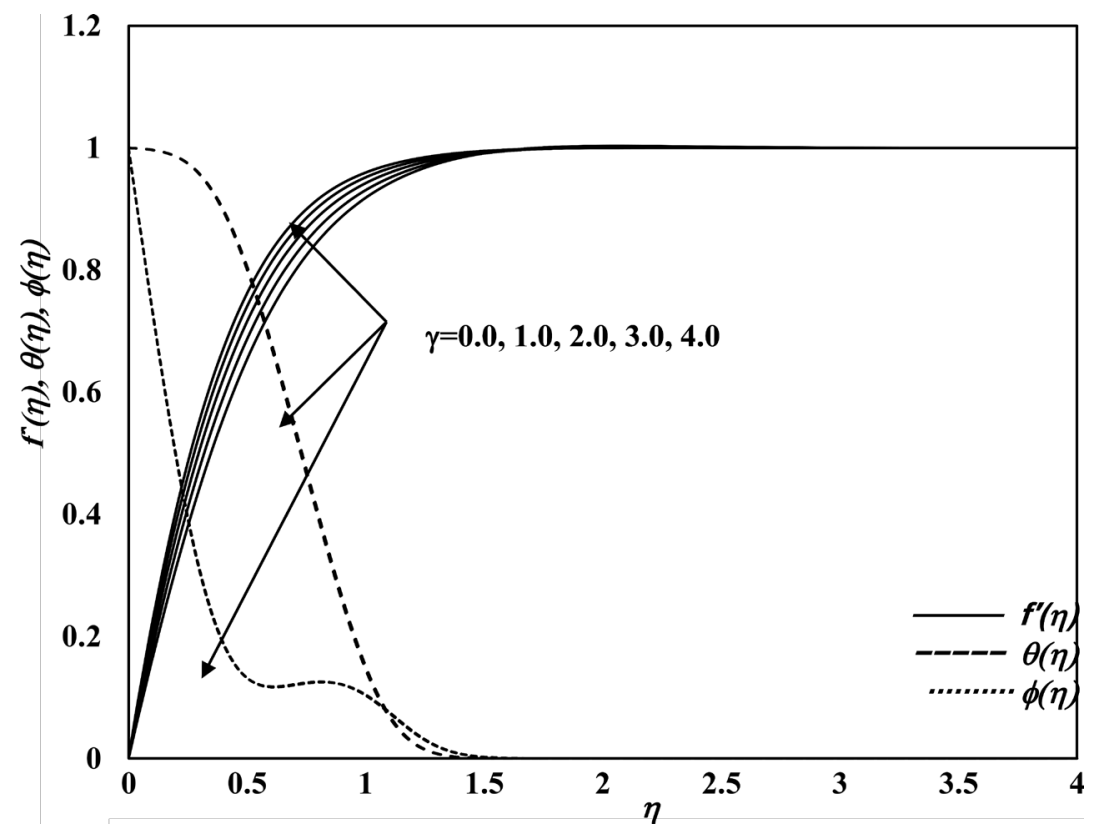

Figure 6. Effects of first resistant parameter $\gamma$ on velocity $f^{\prime}(\eta)$, temperature $\theta(\eta)$ and nanoparticle volume fraction $\phi(\eta)$ with $N_{T}=N_{B}=N_{r}=m=\Delta=0.5, \lambda=1, \operatorname{Pr}=S c=10$ and $\tau=0.5$.

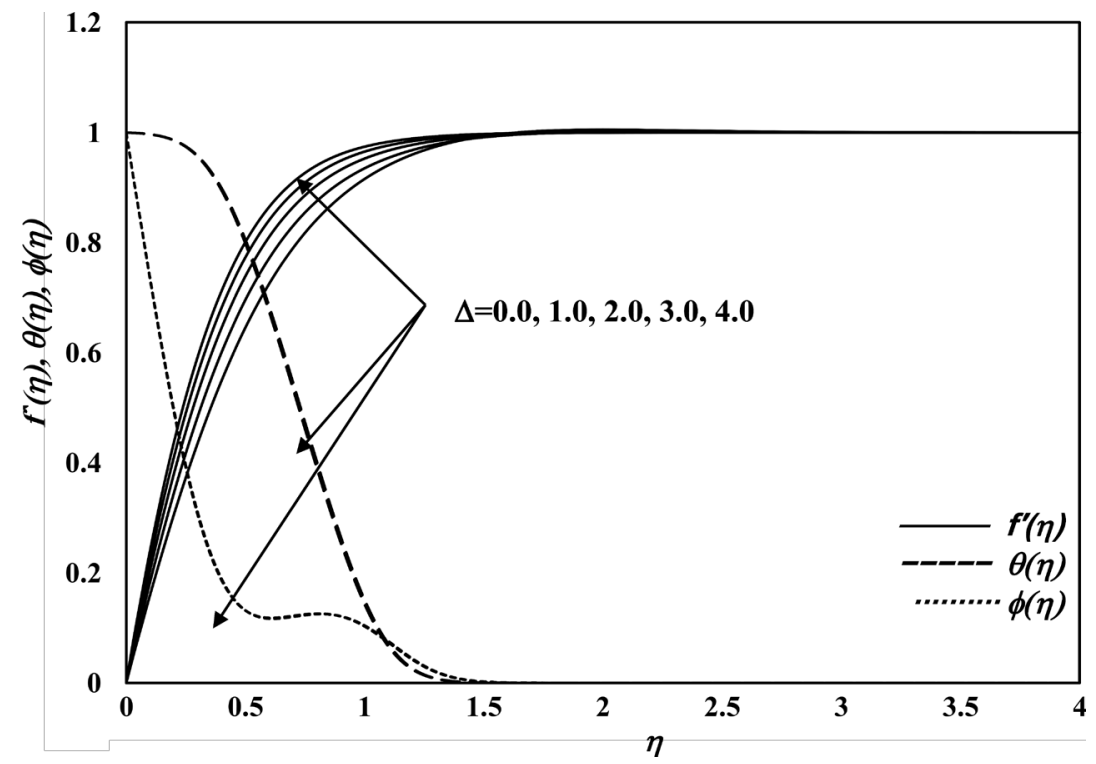

Figure 7. Effects of second resistant parameter $\Delta$ on velocity $f^{\prime}(\eta)$, temperature $\theta(\eta)$ and nanoparticle volume fraction $\phi(\eta)$ with $N_{T}=N_{B}=N_{r}=m=\gamma=0.5, \lambda=1, \operatorname{Pr}=S c=10$ and $\tau=0.5$. 
and nanoparticle volume fraction. An increase in $\gamma$ or $\Delta$ leads to an increase in fluid velocity and a decrease on both temperature and nanoparticle volume fraction. This is due the fact that an increase in $\gamma$ or $\Delta$ implies that there is a decrease in the resistance of the porous medium which tends to accelerate the fluid velocity in the boundary layer region.

Figure 8 displays the effect of Prandtl number Pr on velocity, temperature and nanoparticle volume fraction profiles. It is evident from the figure that, as $\mathrm{Pr}$ increases, velocity profiles have a small increasing. On the other hand, for both temperature and nanoparticle volume fraction profiles, there special points called the "crossing over points" and the temperature and the volume fraction profiles have completely conflicting behavior before and after these points for which the profiles at a fixed value of $\eta$ decreases before that point and increases after it. Prandtl number signifies the ratio of momentum diffusivity to thermal diffusivity. In heat transfer problems, the Prandtl number Pr controls the relative thickening of the momentum and thermal boundary layers. Hence, Prandtl number is small, heat diffuses quickly compared to the velocity (momentum), which means that for liquid metals, the thickness of the thermal boundary layer is much bigger than the momentum boundary layer. Fluids with lower Prandtl number have higher thermal conductivities (and thicker thermal boundary layer structures) so that heat can diffuse from the sheet faster than for higher Pr fluids (thinner boundary layer). So, Prandtl number can be used to increase the rate of cooling in conducting flows.

Figure 9 depicts the effect of Schimdt number $S c$ on velocity, temperature and species concentration profiles. It is noticed that effect of increasing value of $S c$ is

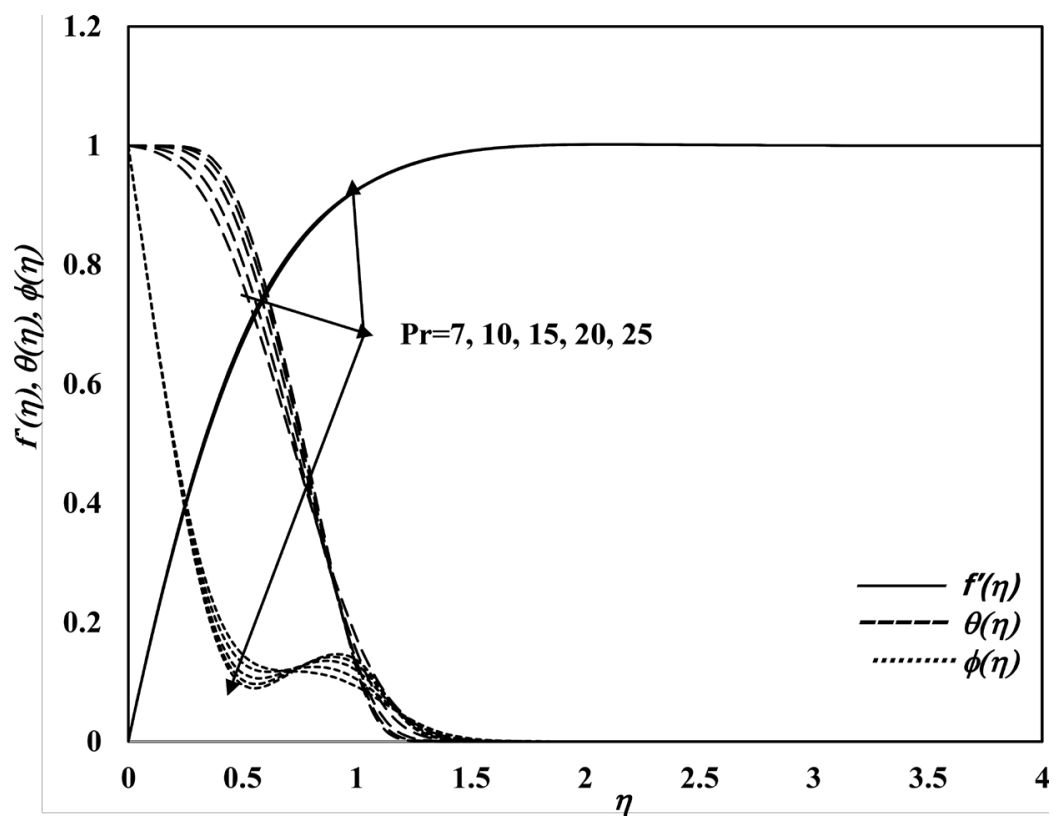

Figure 8. Effects of Prandtl number $\operatorname{Pr}$ on velocity $f^{\prime}(\eta)$, temperature $\theta(\eta)$ and nanoparticle volume fraction $\phi(\eta)$ with $N_{T}=N_{B}=N_{r}=m=\gamma=\Delta=0.5, \lambda=1, S c=10$ and $\tau=0.5$. 


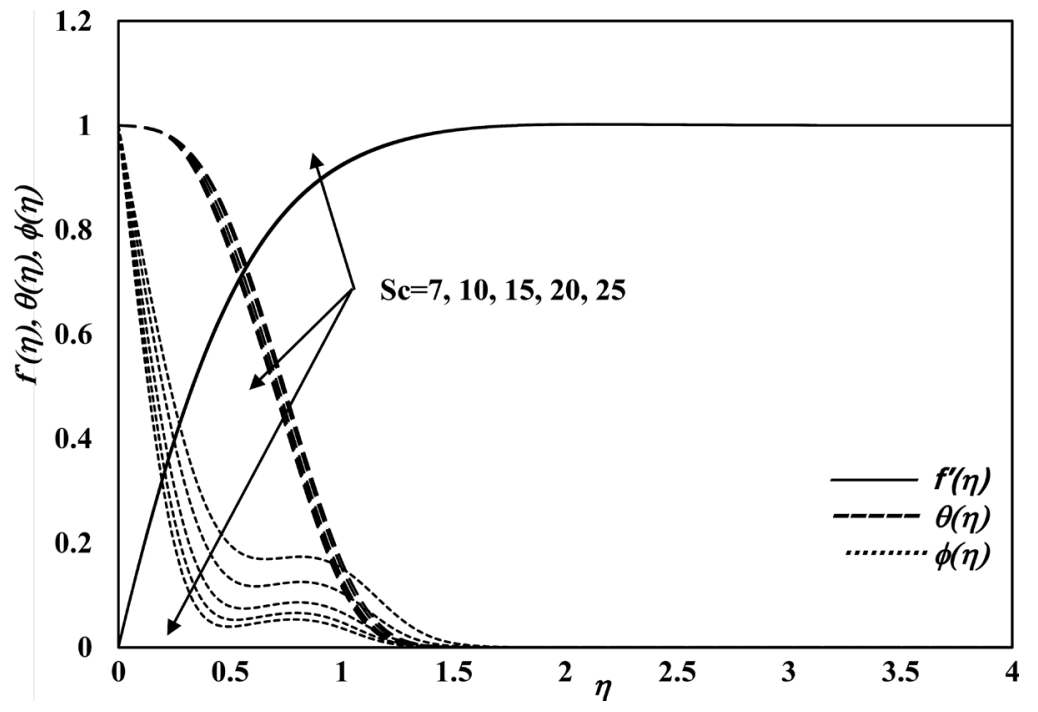

Figure 9. Effects of Schmidt number $S c$ on velocity $f^{\prime}(\eta)$, temperature $\theta(\eta)$ and nanoparticle volume fraction $\phi(\eta)$ with $N_{T}=N_{B}=N_{r}=m=\gamma=\Delta=0.5, \lambda=1, \operatorname{Pr}=10$ and $\tau=0.5$.

to decrease velocity, temperature and species concentration profiles. This is consistent with the fact that, increase in $S c$ means decrease of molecular diffusivity those results in decease of concentration boundary layer. Hence species concentration is higher for small values of $S c$ and lower for large value of $S c$.

Figures 10-17 are presented to illustrate the variations of the local rate of shear stress, the local rate of heat transfer and the local rate of mass transfer for different values of the governing parameters.

Figure 10 display the effects of the thermophoresis parameter $N_{T}$ on the local skin friction coefficient $C_{f}$, local Nusselt number $N u_{x}$ and the local Sherwood number $S h_{x}$. It can be seen that the thermophoresis parameter $N_{T}$ appears in the thermal and the concentration boundary layer equations. As we note that, it is coupled with the temperature function and plays a strong role in determining the diffusion of heat and nanoparticles concentration in the boundary layer. Thus, an increase in the value of the thermophoresis parameter $N_{T}$ leads to a decrease in the local Nusselt number and increases in both the local skin friction coefficient and the local Sherwood number.

Figure 11 display the effects of the Brownian motion parameter $N_{B}$ on the local skin friction coefficient, local Nusselt number and the local Sherwood number. In nanofluid systems, owing to the size of the nanoparticles, Brownian motion takes place, and this can enhance the heat transfer properties. This is due to the fact that the Brownian diffusion promotes heat conduction. The nanoparticles increase the surface area for heat transfer. A nanofluid is a two-phase fluid where the nanoparticles move randomly and increase the energy exchange rates. However, the Brownian motion reduces nanoparticles diffusion. The increase in the local Sherwood number as $N_{B}$ changes is relatively small. Therefore, as indicated before, increasing the value of the Brownian motion parameter $N_{B}$ causes 

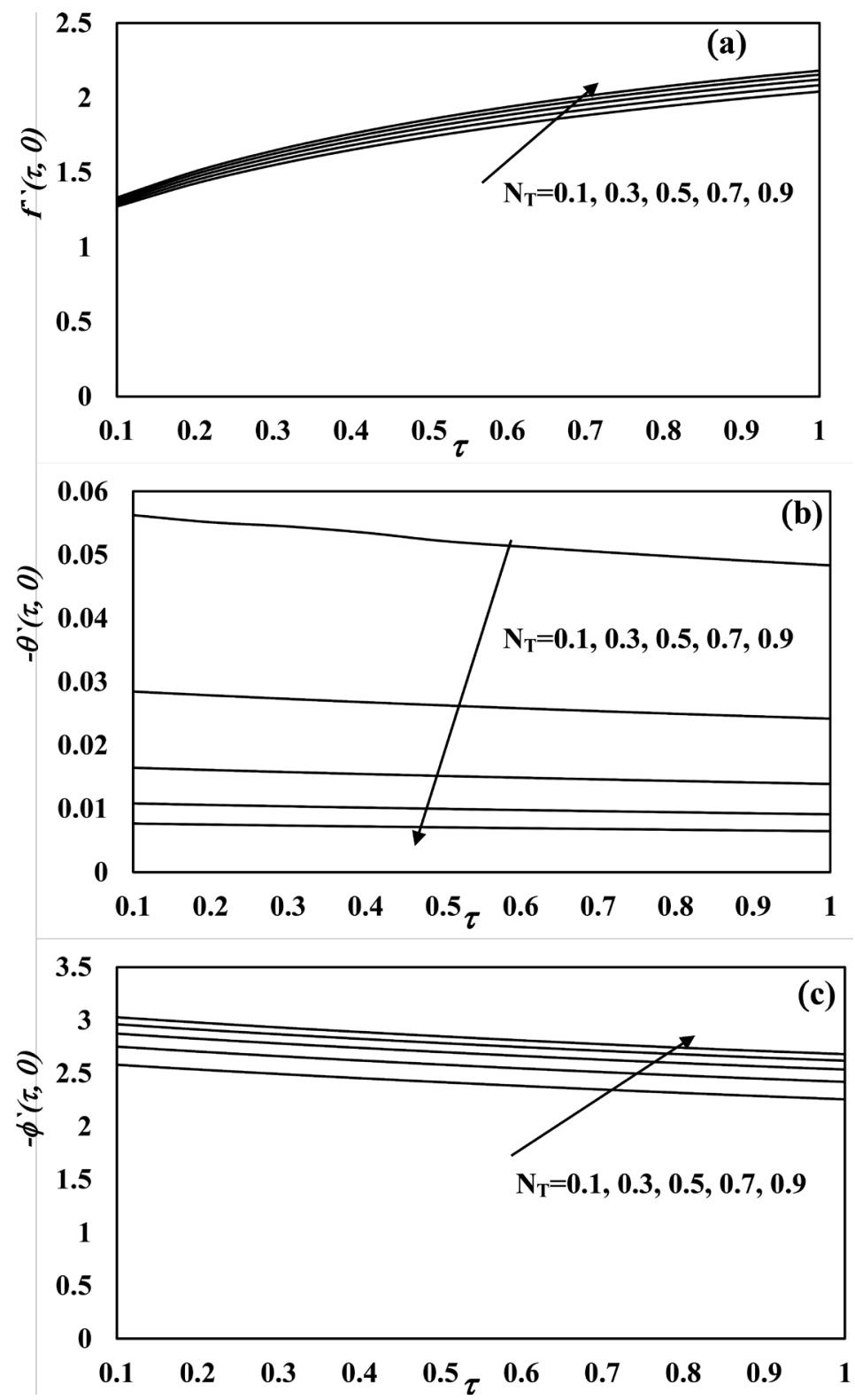

Figure 10. Effects of thermophoresis parameter $N_{T}$ on (a) skin fraction, (b) Nusselt number and (c) Sherwood number with $N_{r}=N_{B}=\gamma=\Delta=0.5, m=1, \operatorname{Pr}=S c=10$ and $\lambda=1$.

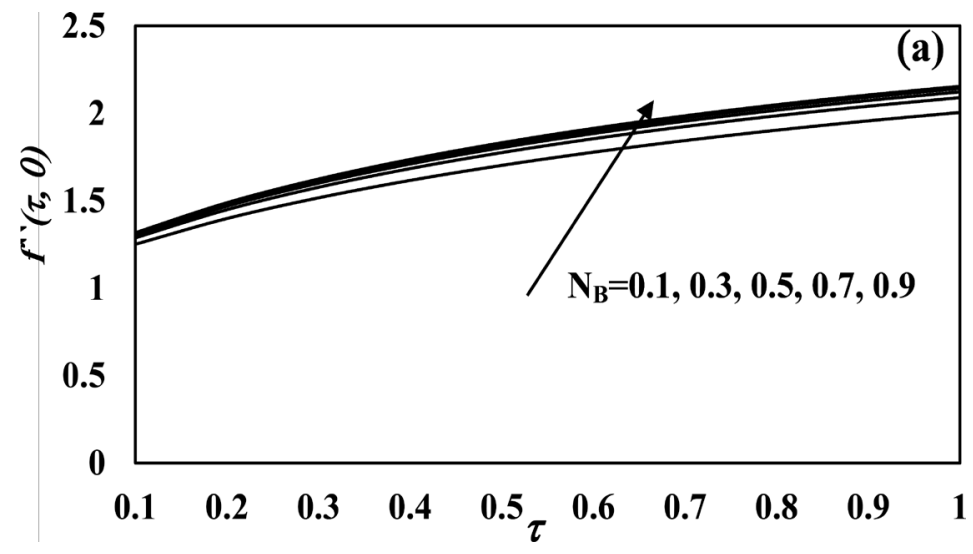



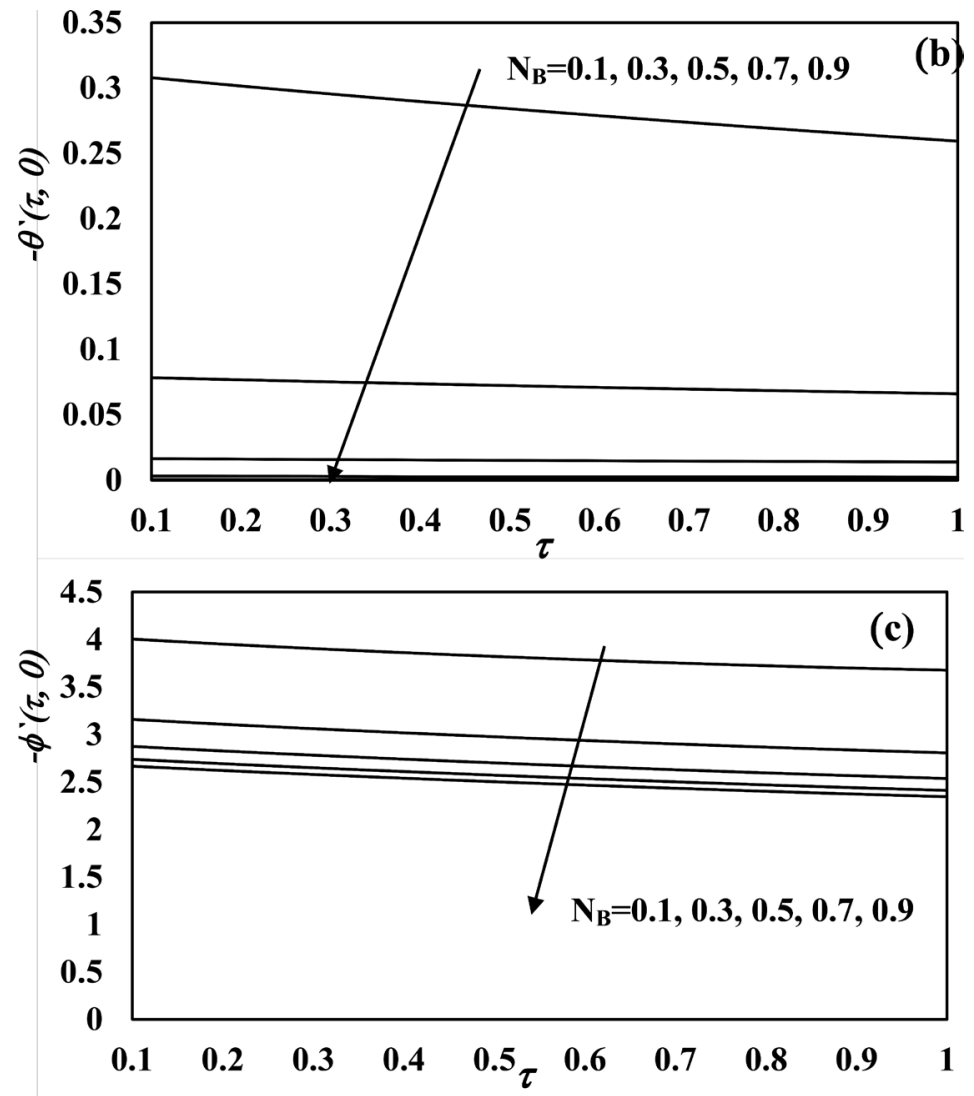

Figure 11. Effects of Brownian motion parameter $N_{B}$ on (a) skin fraction, (b) Nusselt number and (c) Sherwood number with $N_{r}=N_{T}=\gamma=\Delta=0.5, m=1, \operatorname{Pr}=S c=10$ and $\lambda=1$.
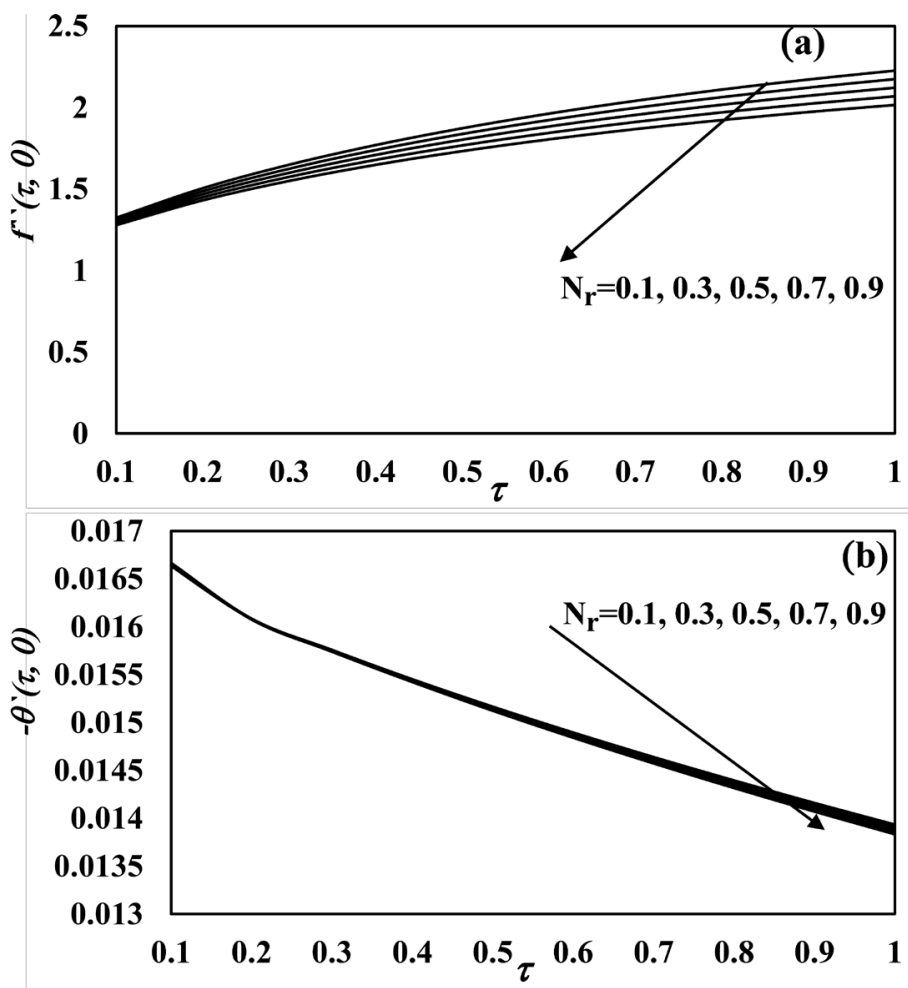


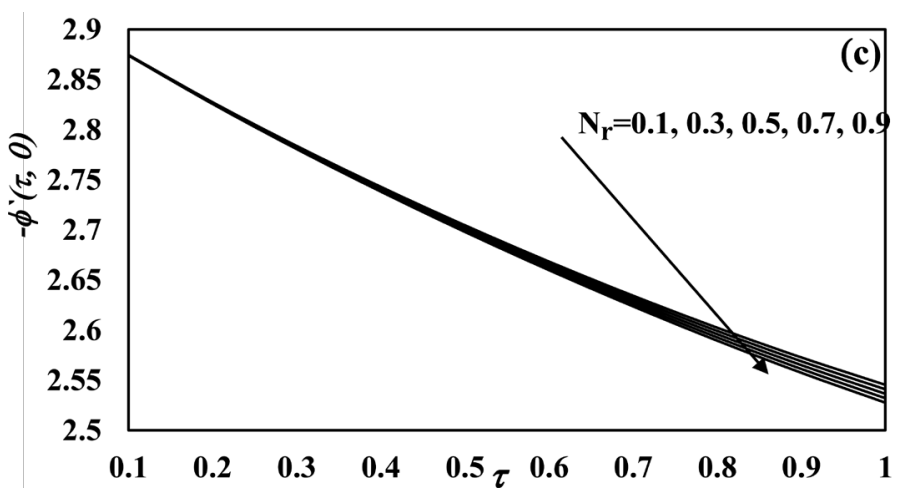

Figure 12. Effects of buoyancy ratio parameter $N_{r}$ on (a) skin fraction, (b) Nusselt number and (c) Sherwood number with $N_{T}=N_{B}=\gamma=\Delta=0.5, m=1, \operatorname{Pr}=S c=10$ and $\lambda=1$.
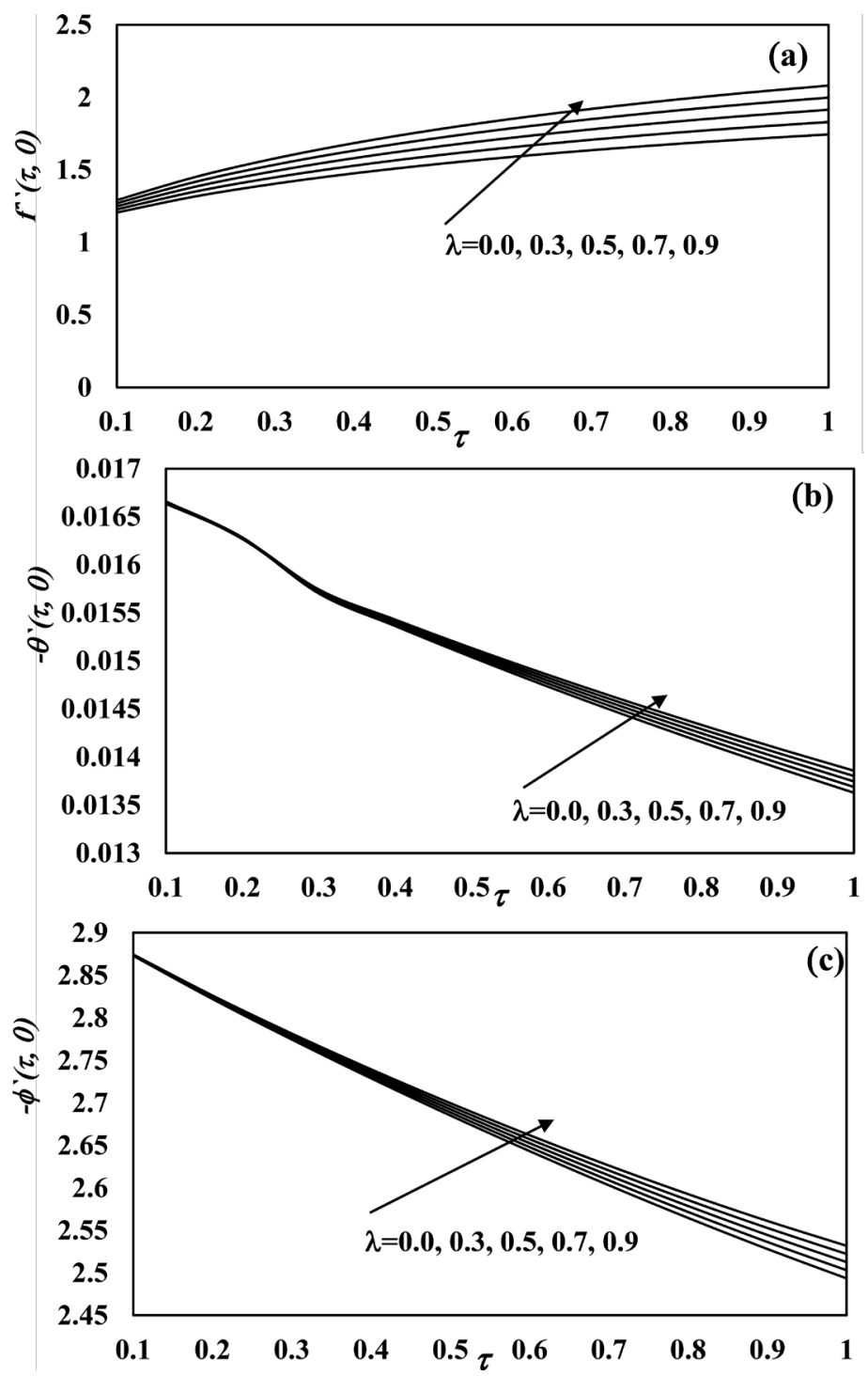

Figure 13. Effect of mixed convection parameter $\lambda$ on (a) skin fraction, (b) Nusselt number and (c) Sherwood number with $N_{r}=N_{T}=N_{B}=\gamma=\Delta=0.5, m=1, \operatorname{Pr}=10$ and $S c=10$. 

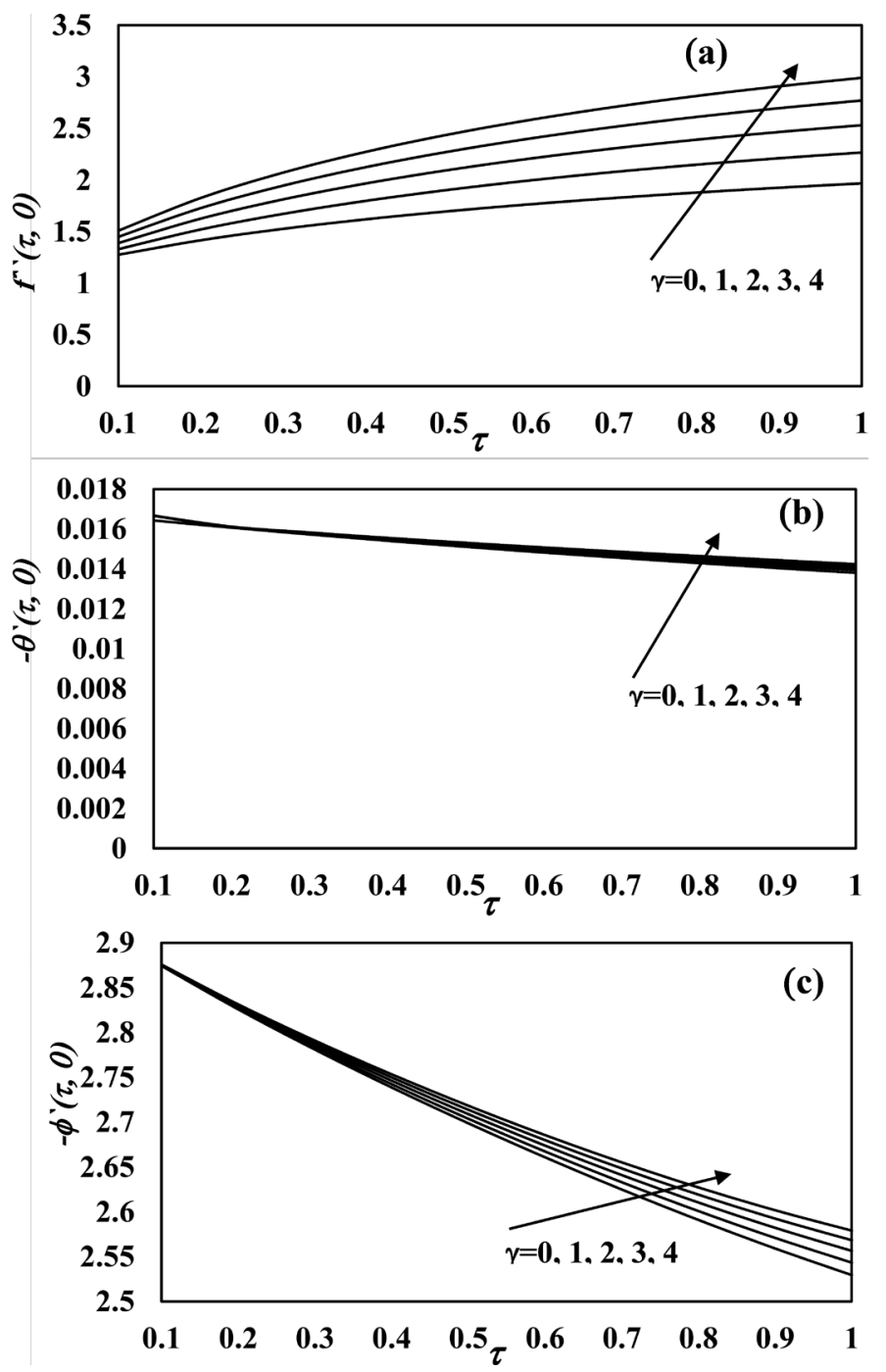

Figure 14. Effects of first resistant parameter $\gamma$ on (a) skin fraction, (b) Nusselt number and (c) Sherwood number with $N_{r}=N_{T}=\Delta=0.5, m=1, \operatorname{Pr}=S c=10$ and $\lambda=1$.

increases in the velocity and temperature profiles while its volume fraction profile decreases. This yields reductions in both the local Nusselt number and the local Sherwood number and enhancement in the local skin-friction coefficient.

The effects of the buoyancy ratio parameter $N_{r}$ are illustrated in Figure 12. It is observed from this figure that an increase in the value of $N_{r}$ tends to decrease all of the local skinfriction coefficient, the local Nusselt number and the local Sherwood number.

The effects of mixed convection parameter $\lambda$ on skin friction coefficient, local Nusselt number and local Sherwood number are are shown in Figure 13. It is observed that as $\lambda$ increases all the skin friction coefficient, local Nusselt number and local Sherwood number increases. The physical reason is that the positive buoyancy force $(\lambda>0)$ implies favorable pressure gradient, and the fluid gets accelerated, which results in thinner momentum boundary layer. Consequently, the local skin friction is also increased at all times. 

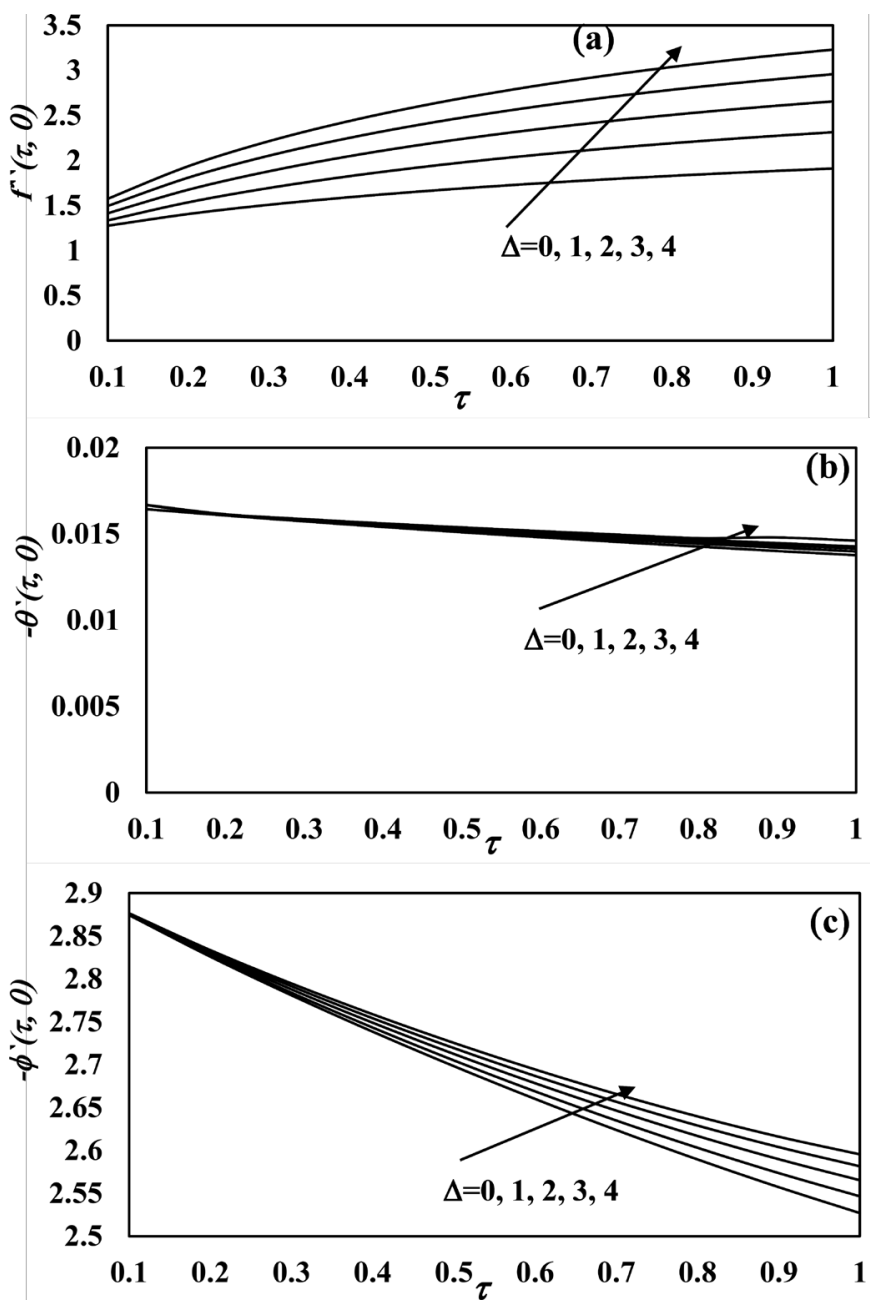

Figure 15. Effects of second resistant parameter $\Delta$ on (a) skin fraction, (b) Nusselt number and (c) Sherwood number with $N_{r}=N_{T}=N_{B}=\gamma=0.5, m=1, \operatorname{Pr}=S c=10$ and $\lambda=1$.

The effects of both the first resistance parameter $\gamma$ and the second resistance parameter $\Delta$ on the local skin-friction coefficient, the local Nusselt number and the local Sherwood number are illustrated in Figure 13 and Figure 14, respectively. From these figures, it is observed that both $\gamma$ and $\Delta$ have the same behavior. From these figures, we conclude that both the first and second resistances enhance all of the local rate of shear stress (the local skin-friction coefficient), the local rate of heat transfer (local Nusselt number) and the local rate of mass transfer (local Sherwood number).

The effects of Prandtl number Pr and Schmidt number $S c$ on the behaviors of the local skin-friction coefficient, local Nusselt number and the local Sherwood number are illustrated on Figure 16 and Figure 17, respectively. It is observed from these figures that increasing the value of $\operatorname{Pr}$ or $S c$ produces increases in the local rate of shear stress and decreases in both of the heat transfer rate and the mass transfer rate. This is associated with the decreases in the nanoparticle boundary layer as $S c$ increases as discussed earlier. 

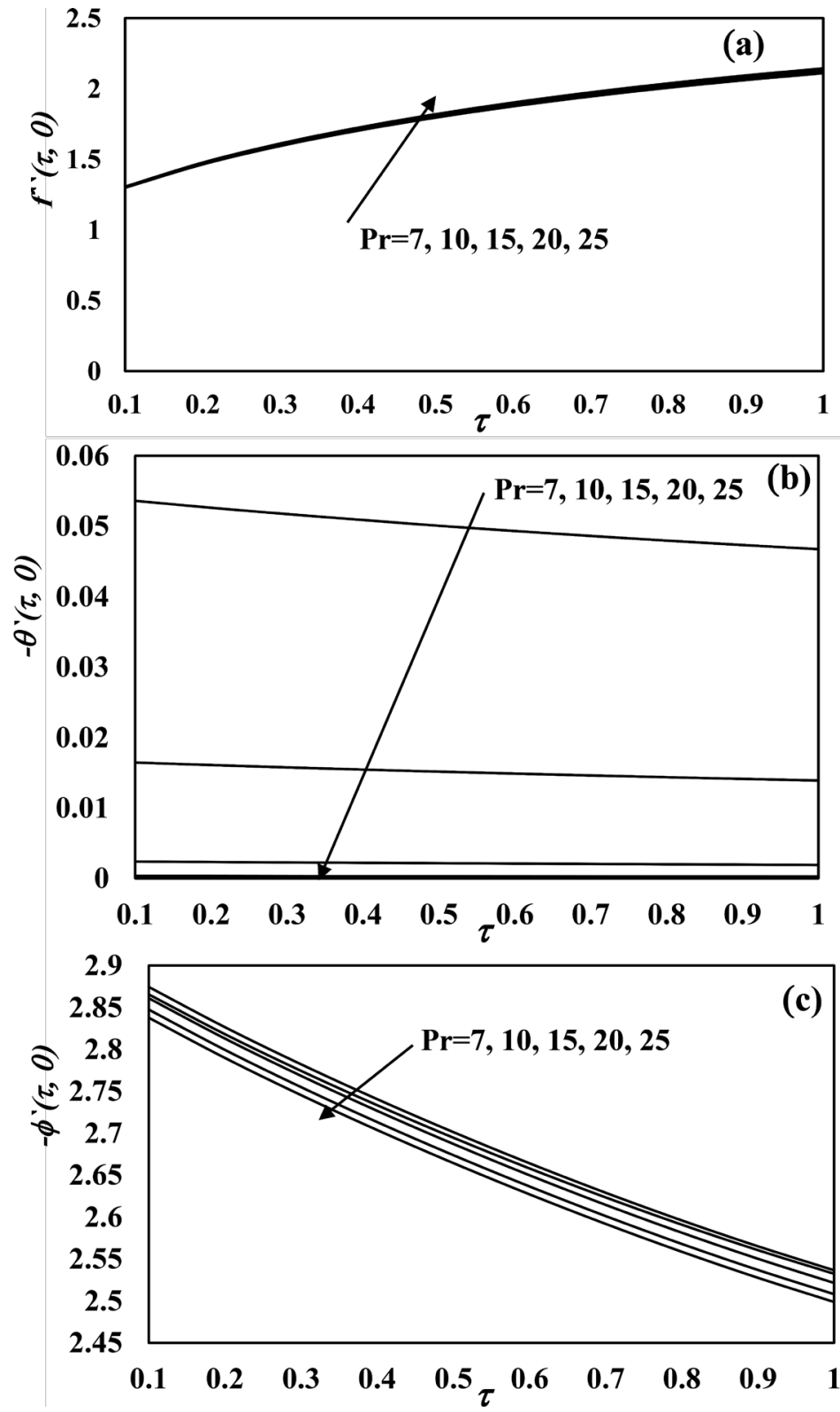

Figure 16. Effects of Prandtl number Pr on (a) skin fraction, (b) Nusselt number and (c) Sherwood number with $N_{r}=N_{T}=N_{B}=\gamma=\Delta=0.5, m=1, S c=10$ and $\lambda=1$.

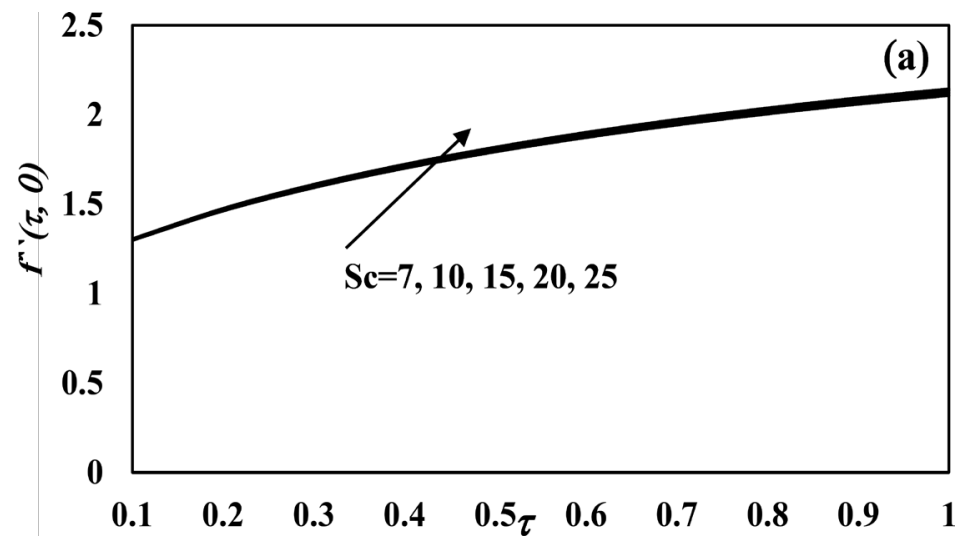



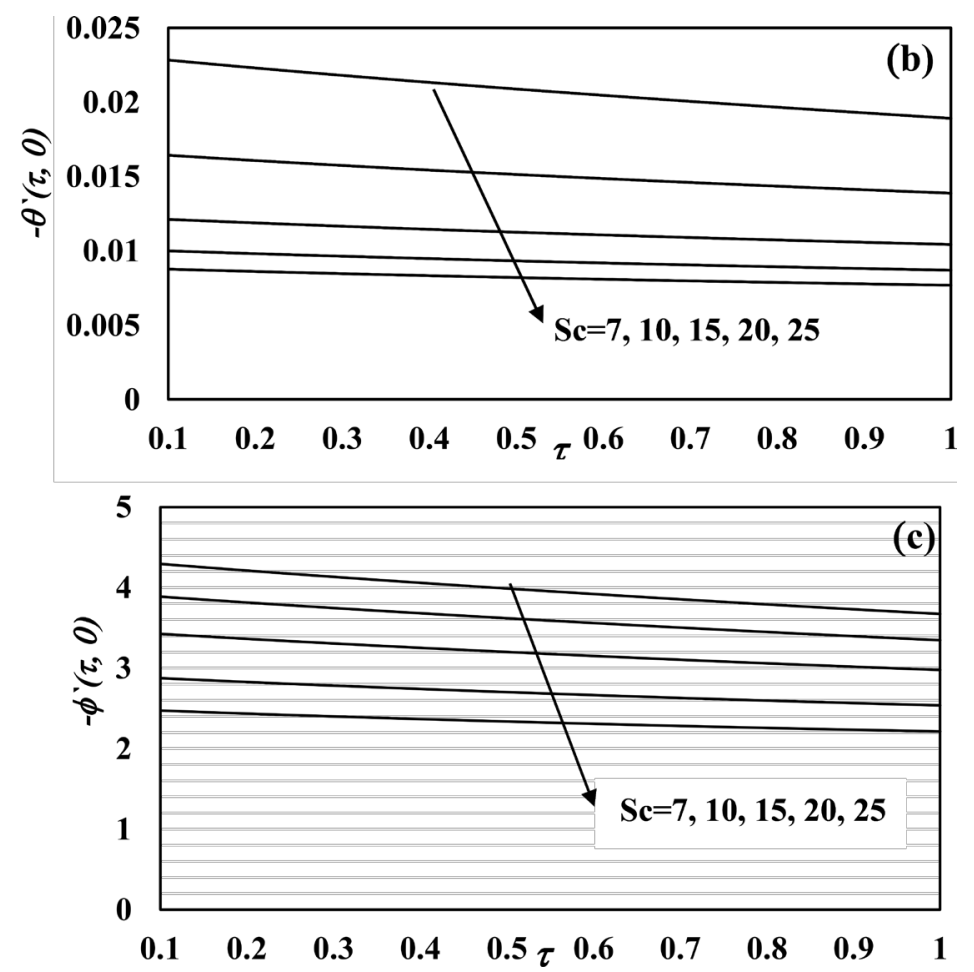

Figure 17. Effects of Schimdt number $S c$ on (a) skin fraction, (b) Nusselt number and (c) Sherwood number with $N_{r}=N_{T}=N_{B}=\gamma=\Delta=0.5, m=1, \operatorname{Pr}=10$ and $\lambda=1$.

Finally, from Figures 10-17, it is observed that as the unsteadiness parameter $\tau$ increases the local rate of shear stress increases while, both of the local rate of heat transfer and the local rate of mass transfer decreases.

\section{Conclusions}

In the present work, the problem of unsteady mixed convection flow along a symmetric wedge embedded in a porous medium saturated with a nanofluidis studied theoretically. The resulting system of nonlinear partial differential equations is treated using the Sparrow-Quack-Boerner local non-similarity numerical method. The obtained system is solved numerically using an efficient numerical shooting technique with a fourth-fifth-order Runge-Kutta method scheme (MATLAB package). The solutions for the flow and the heat and mass transfer characteristics are evaluated numerically for various values of the governing parameters, namely the unsteadiness parameter $\tau$, mixed convection parameter $\lambda$, nanofluid buoyancy ratio parameter $N_{r}$, thermophoresis parameter $N_{T}$, Brownian motion parameter $N_{B}$ first resistance parameter $\gamma$, second resistance parameter $\Delta$, Prandtl number $P r$ and the Schmidt number $S c$. The following are brief summary conclusions drawn from the analysis:

1) The thickness of the momentum boundary layer slightly decreases with an increase in the nanofluid buoyancy ratio parameter $N_{r}$ However, it increases with the increase of all other parameters.

2) The thickness of the thermal boundary layer increases with an increase in 
both of $N_{T}$ and $N_{B}$ parameters while it slightly increases with an increase in $N_{r}$ parameter.

3) The nanoparticle volume fraction boundary-layer thickness decreases obviously with an increase in both $N_{T}$ and $N_{B}$ parameters and slightly increases with an increase in the $N_{r}$ parameter.

4) The buoyancy parameter $\lambda$ enhances obviously the momentum boundarylayer thickness and slightly reduce both of the thermal and the nanoparticle volume fraction boundary-layer thicknesses.

5) Both the first and the second resistance parameters $\gamma$ and $\Delta$ enhance the momentum boundary-layer thickness and reduce both of the thermal and the nanoparticle volume fraction boundary-layer thicknesses.

6) The magnitude of the skin-friction coefficient $f^{\prime \prime}(0)$ decreases with increasing values of the nanofluid buoyancy ratio parameter $N_{r}$ and increases with the increase of all other parameters.

7) The local Nusselt number decreases with all the nanofluid parameters $N_{T}$, $N_{B}$ and $N_{r}$. While, it decreases with increases in the Prandtl number $P r$ and the Schmidt number $S c$.

8) The local Sherwood number increases as the $N_{T}$ parameter increases. However, it decreases as either of $N_{B}, N_{r}$, Pror $S c$ increases.

9) The unsteadiness parameter $\tau$ and the resistance parameters $\gamma$ and $\Delta$ enhance the local skin-friction coefficient, local Nusselt number and the local Sherwood number.

\section{Acknowledgements}

The authors would like to thank Institute of Scientific Research and Revival of Islamic Heritage at the Umm Al-Qura University (Project ID No. 43405010) for the financial support.

\section{Conflicts of Interest}

The authors declare no conflicts of interest regarding the publication of this paper.

\section{References}

[1] Smith, S.H. (1967) The Impulsive Motion of a Wedge in a Viscous Fluid. Zeitschrift für Angewandte Mathematik und Physik, 18, 508-522. https://doi.org/10.1007/BF01601721

[2] Nanbu, K. (1969) Unsteady Falkner-Skan Flow. Zeitschrift für Angewandte Mathematik und Physik, 22, 1167-1172. https://doi.org/10.1007/BF01590885

[3] Hall, M.G. (1969) The Boundary-Layer over an Impulsively Started Flat Plate. Proceedings of the Royal Society of London, Series A, 310, 401-414. https://doi.org/10.1098/rspa.1969.0083

[4] Harris, D.S., Ingham, D.B. and Pop, I. (2002) Unsteady Heat Transfer in Impulsive Falkner-Skan Flows: Constant Wall Temperature Case. European Journal of Mechanics-B/ Fluids, 21, 447-468. https://doi.org/10.1016/S0997-7546(02)01193-7 
[5] Keller, H.B. and Cebeci, T. (1971) Accurate Numerical Methods for BoundaryLayer Flows. Part 1. Two-Dimensional Laminar Flows. Proceedings of the 2nd International Conference on Numerical Methods in Fluid Dynamics, Berkeley, 15-19 September 1970, 92-100. https://doi.org/10.1007/3-540-05407-3_15

[6] Hossain, M.A. (1992) Simultaneous Heat and Mass Transfer on Oscillatory Free Convection Boundary-Layer Flow. International Journal of Energy Research, 12, 205 216. https://doi.org/10.1002/er.4440120203

[7] Hossain, M.A. (1992) Effect of Transpiration on Combined Heat and Mass Transfer in Mixed Convection along a Vertical Plate. International Journal of Energy Research, 16, 761-769. https://doi.org/10.1002/er.4440160812

[8] Hossain, M.A., Banu, N. and Nakayama, A. (1994) Non-Darcy Forced Convection Boundary-Layer Flow over a Wedge Embedded in a Saturated Porous Medium. $\mathrm{Nu}$ merical Heat Transfer, Part A: Applications, 26, 399-414.

https://doi.org/10.1080/10407789408956000

[9] Watkins, C.B. (1976) Unsteady Heat Transfer in Impulsive Falkner-Skan Flows. International Journal of Heat and Mass Transfer, 19, 395-403.

https://doi.org/10.1016/0017-9310(76)90095-8

[10] Williams, J.C. and Rhyne, T.B. (1980) Boundary-Layer Development on a Wedge Impulsively Set into Motion. SIAM Journal on Applied Mathematics, 38, 215-224. https://doi.org/10.1137/0138019

[11] Ibrahim, F.S., Hassanien, I.A. and Bakr, A.A. (2004) Unsteady MHD Micropolar Fluid Flow and Heat Transfer over a Vertical Porous Plate through Porous Medium in the Presence of Thermal and Mass Diffusion with Constant Heat Source. Canadian Journal of Physics, 82, 775-790. https://doi.org/10.1139/p04-021

[12] Hassanien, I.A., Ibrahim, F.S. and Omer, G.M. (2004) Unsteady Free Convection Flow in the Stagnation-Point Region of a Rotating Sphere Embedded in a Porous Medium. Mechanics and Mechanical Engineering, 7, 89-98.

[13] Ibrahim, F.S. (2008) Unsteady Mixed Conviction Flow in the Stagnation Region of a Three Dimensional Body Embedded in a Porous Medium. Nonlinear Analysis. Modeling and Control, 13, 31-46. https://doi.org/10.15388/NA.2008.13.1.14587

[14] Al-Harbi, S.M. and Hassanien, I.A. (2011) Unsteady Mixed Convection Flow in the Stagnation Region of a Heated Vertical Plate Embedded in a Variable Porosity Medium with Thermal Dispersion Effects. In: Dos Santos Bernardes, M.A., Ed., Developments in Heat Transfer, IntechOpen, London.

[15] Al-Harbi, S.M. (2011) Self-Similar Solution of Heat and Mass Transfer of Unsteady Mixed Convection Flow on a Rotating Cone Embedded in a Porous Medium Saturated with a Rotating Fluid. Applied Mathematics, 2, 1196-1203.

http://dx.doi.org/10.4236/am.2011.210166

[16] Sameh, E. A, and Rashed, Z.Z., 2018, Unsteady MHD Mixed Convection Flow with Slip of a Nanofluid in the Stagnation Region of an Impulsively Rotating Sphere with Effects of Thermal Radiation and Convective Boundary Conditions. World Journal of Mechanics, 8, 137-160. https://doi.org/10.4236/wjm.2018.85011

[17] Pop, I. and Ingham, D.B. (2001) Convective Heat Transfer: Mathematical and Computational Modeling of Viscous Fluids and Porous Media. Pergamon, Oxford.

[18] Nield, D.A. and Bejan, A. (2005) Convection in Porous Media. Springer-Verlag, New York.

[19] Choi, S.U.S. (1995) Enhancing Thermal Conductivity of Fluid with Nanoparticles, Developments and Applications of Non-Newtonian FED-Vol. 231/MD-Vol. 66, ASME, New York, 99-105. 
[20] Khanafer, K., Vafai, K. and Lightstone, M. (2003) Buoyancy-Driven Heat Transfer Enhancement in a Two-Dimensional Enclosure Utilizing Nanofluids. International Journal of Heat and Mass Transfer, 46, 3639-3653. https://doi.org/10.1016/S0017-9310(03)00156-X

[21] Nnanna, A.G.A. (2007) Experimental Model of Temperature-Driven Nanofluid. Journal of Heat Transfer, 129, 697-704. https://doi.org/10.1115/1.2717239

[22] Oztop, H.F. and Abu-Nada, E. (2008) Numerical Study of Natural Convection in Partially Heated Rectangular Enclosures Filled with Nanofluids. International Journal of Heat and Fluid Flow, 29, 1326-1336. https://doi.org/10.1016/j.ijheatfluidflow.2008.04.009

[23] Aminossadati, S.M. and Ghasemi, B. (2009) Natural Convection Cooling of a Localised Heat Source at the Bottom of a Nanofluid-Filled Enclosure. European Journal of Mechanics-B/ Fluids, 28, 630-640. https://doi.org/10.1016/j.euromechflu.2009.05.006

[24] Yacob, N., Ishak A. and Pop I. (2011) Falkner-Skan Problem for a Static or Moving Wedge in Nanofluids. International Journal of Thermal Sciences, 50, 133-139. https://doi.org/10.1016/j.ijthermalsci.2010.10.008

[25] Gorla, R.S.R., Chamkha, A.J. and Rashad, A.M. (2011) Mixed Convective Boundary Layer Flow over a Vertical Wedge Embedded in a Porous Medium Saturated with a Nanofluid: Natural Convection Dominated Regime. Nanoscale Research Letters, 6, Article No. 207. https://doi.org/10.1186/1556-276X-6-207

[26] Hamad, M., Pop, I. and Ismail, A. (2011) Magnetic Field Effects on Free Convection Flow of a Nanofluid Past a Semi-Infinite Vertical Flat Plate. Nonlinear Analysis. Real World Applications, 12, 1338-1346. https://doi.org/10.1016/j.nonrwa.2010.09.014

[27] Buongiorno, J. (2006) Convective Transport in Nanofluids. Journal of Heat Transfer, 128, 240-250. https://doi.org/10.1115/1.2150834

[28] Hamad, M. (2011) Analytical Solution of Natural Convection Flow of a Nanofluid over a Linearly Stretching Sheet in the Presence of Magnetic Field. International Communications in Heat and Mass Transfer, 38, 487-492. https://doi.org/10.1016/j.icheatmasstransfer.2010.12.042

[29] Khan, W. and Pop, I. (2010) Boundary-Layer Flow of a Nanofluid Past a Stretching Sheet. International Journal of Heat and Mass Transfer, 53, 2477-2483. https://doi.org/10.1016/j.ijheatmasstransfer.2010.01.032

[30] Abu-Nada, E. and Chamkha, A. (2010) Effect of Nanofluid Variable Properties on Natural Convection in Enclosures Filled with a CuO-EG-Water Nanofluid. International Journal of Thermal Sciences, 49, 2339-2352. https://doi.org/10.1016/j.ijthermalsci.2010.07.006

[31] Das, S., Choi, S., Yu, W. and Pradeep, T. (2007) Nanofluids: Science and Technology. Wiley, New Jersey. https://doi.org/10.1002/9780470180693

[32] Kakaç, S. and Pramuanjaroenkij, A. (2009) Review of Convective Heat Transfer Enhancement with Nanofluids. International Journal of Heat and Mass Transfer, 52, 3187-3196. https://doi.org/10.1016/j.ijheatmasstransfer.2009.02.006

[33] Muthtamilselvan, M., Kandaswamy, P. and Lee, J. (2010) Heat Transfer Enhancement of Copper-Water Nanofluids in a Lid-Driven Enclosure. Communications in Nonlinear Science and Numerical Simulation, 15, 1501-1510. https://doi.org/10.1016/j.cnsns.2009.06.015

[34] Hady, F.M., Ibrahim, F.S., El-Hawary, H.M.H. and Abdelhady, A.M. (2012) Forced Convection Flow of Nanofluids Past Power Law Stretching Horizontal Plates. Ap- 
plied Mathematics, 3, 121-126. http://dx.doi.org/10.4236/am.2012.32019

[35] Hady, F.M., Ibrahim, F.S., El-Hawary, H.M.H. and Abdelhady, A.M. (2012) Effect of Suction/Injection on Natural Convective Boundary-Layer Flow of a Nanofluid Past a Vertical Porous Plate through a Porous Medium. Journal of Modern Methods in Numerical Mathematics, 3, 53-63.

[36] Hady, F.M., Ibrahim, F.S., Abdel-Gaied, S.M. and Eid, M.R. (2012) Radiation Effect on Viscous Flow of a Nanofluid and Heat Transfer over a Nonlinearly Stretching Sheet. Nanoscale Research Letters, 7, Article No. 229.

https://doi.org/10.1186/1556-276X-7-229

[37] James, M., Mureithi, E.W. and Kuznetsov, D. (2015) Effects of Variable Viscosity of Nanofluid Flow over a Permeable Wedge Embedded in Saturated Porous Medium with Chemical Reaction and Thermal Radiation. International Journal of Advances in Applied Mathematics and Mechanics, 2, 101-118.

[38] Uddin, M., Alim, M. and Rahman, M. (2019) MHD Effects on Mixed Convective Nanofluid Flow with Viscous Dissipation in Surrounding Porous Medium. Journal of Applied Mathematics and Physics, 7, 968-982.

https://doi.org/10.4236/jamp.2019.74065

[39] Chamkha, A.J., Nabwey, H.A., Abdelrahman, Z.M.A. and Rashad, A.M. (2019) Mixed Bioconvective Flow over a Wedge in Porous Media Drenched with a Nanofluid. Journal of Nanofluids, 9, 24-35. https://doi.org/10.1166/jon.2020.1728

[40] Lai, F.C. (2000) Mixed Convection in Saturated Porous Media. In: Vafai, K., Ed., Handbook of Porous Media, Marcel Dekker, New York, 605-661.

[41] Pereyra, V. (1978) PASVA3, an Adaptive Finite Difference Fortran Program for First Order Non-Linear Boundary Value Problems. In: Childs, B., Scott, M., Daniel, J.W., Denman, E., Nelson, P., Eds., Codes for Boundary-Value Problems in Ordinary Differential Equations, Vol. 76, Springer, Berlin, Heidelberg. https://doi.org/10.1007/3-540-09554-3_4

[42] Scshadri, R., Srccshylan, N. and Nath, G. (2002) Unsteady Mixed Convection Flow in the Stagnation Region of a Heated Vertical Plate Due to Impulsive Motion. International Journal of Heat and Mass Transfer, 45, 1345-1352.

https://doi.org/10.1016/S0017-9310(01)00228-9 